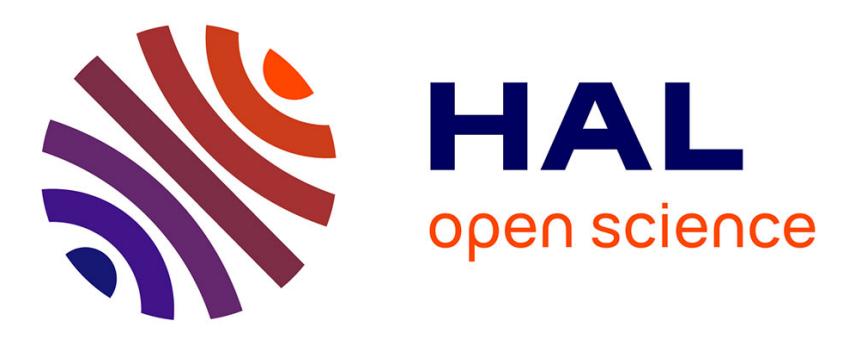

\title{
The Fundamental Theorem of Asset Pricing Under Transaction Costs
}

\author{
Paolo Guasoni, Emmanuel Lépinette, Miklos Rasonyi
}

\section{To cite this version:}

Paolo Guasoni, Emmanuel Lépinette, Miklos Rasonyi. The Fundamental Theorem of Asset Pricing Under Transaction Costs. Finance and Stochastics, 2012, pp.Online First. 10.1007/s00780-012-01850 . hal-00700844

\section{HAL Id: hal-00700844 https://hal.science/hal-00700844}

Submitted on 24 May 2012

HAL is a multi-disciplinary open access archive for the deposit and dissemination of scientific research documents, whether they are published or not. The documents may come from teaching and research institutions in France or abroad, or from public or private research centers.
L'archive ouverte pluridisciplinaire HAL, est destinée au dépôt et à la diffusion de documents scientifiques de niveau recherche, publiés ou non, émanant des établissements d'enseignement et de recherche français ou étrangers, des laboratoires publics ou privés. 


\title{
BOSTON UNIVERSITY
}

\section{Boston University School of Management}

\author{
Boston University School of Management Research Paper Series
}

No. 2011-7

"The Fundamental Theorem of Asset Pricing under Transaction Costs"

\author{
Emmanuel Denis \\ Paolo Guasoni \\ Miklós Rásonyi
}

Electronic copy available at: http://ssrn.com/abstract=1784440 


\title{
The Fundamental Theorem of Asset Pricing under Transaction Costs
}

\author{
Emmanuel Denis · Paolo Guasoni · Miklós \\ Rásonyi
}

the date of receipt and acceptance should be inserted later

\begin{abstract}
This paper proves the Fundamental Theorem of Asset Pricing with transaction costs, when bid and ask prices follow locally bounded càdlàg (right-continuous, left-limited) processes.

The Robust No Free Lunch with Vanishing Risk (RNFLVR) condition for simple strategies is equivalent to the existence of a strictly consistent price system (SCPS). This result relies on a new notion of admissibility, which reflects future liquidation opportunities. The (RNFLVR) condition implies that admissible strategies are predictable processes of finite variation.

The appendix develops an extension of the familiar Stieltjes integral for càdlàg integrands and finite-variation integrators, which is central to modeling transaction costs with discontinuous prices.
\end{abstract}

Mathematics Subject Classification (2000) Primary 91B28 · Secondary 62P05, 26A $45,60 \mathrm{H} 05$

Keywords Arbitrage · Fundamental Theorem of Asset Pricing · Transaction Costs · Admissible Strategies · Finite Variation

We are indebted to Walter Schachermayer for many stimulating discussions which inspired this paper. We also thank Friedrich Hubalek for useful comments.

Supported by NSF (DMS-053239 and DMS-0807994), the Hungarian Science Foundation (OTKA, F 049094), and the Austrian Science Fund (FWF, P 19456), at the Financial and Actuarial Mathematics Research Unit of Vienna University of Technology.

Paris Dauphine University, Ceremade, Place du Marchal De Lattre De Tassigny, 75775 Paris Cedex 16, France, E-mail: emmanuel.denis@ ceremade.dauphine.fr · Boston University, Department of Mathematics and Statistics, 111 Cummington St, Boston, MA 02215, United States · Dublin City University, School of Mathematical Sciences, Glasnevin, Dublin 9, Ireland E-mail: guasoni@bu.edu · School of Mathematics, University of Edinburgh, Kings Buildings, Edinburgh, EH9 3JZ, United Kingdom · MTA SZTAKI, Institute for Computer Science and Control, Budapest · Pazmany Peter Catholic University, Budapest. 


\section{Introduction}

The equivalence between absence of arbitrage and the existence of a positive linear pricing rule is central to Finance, and was pioneered by Ross (1977), in the wake of the Black \& Scholes (1973) option pricing model. Dybvig \& Ross (1987) referred to such results as "The Fundamental Theorem of Asset Pricing" (FTAP), setting the tone for today's terminology.

Thirty years later, the Fundamental Theorem remains an active research topic for markets with frictions, and a practically oriented reader may look with skepticism at another paper on this subject. Yet, there is a reason for this ongoing interest: the path to the FTAP leads to the natural notions of admissibility and arbitrage, which underlie all practical applications. This is a major lesson of the work of Delbaen \& Schachermayer (1994) in frictionless markets. The Fundamental Theorem seems inevitable.

This paper proves a version of the FTAP in continuous time, for a risky asset driven by a càdlàg and locally bounded bid-ask process. The main result is the equivalence between a no-arbitrage condition, the Robust No Free Lunch with Vanishing Risk (RNFLVR), and the existence of a Strictly Consistent Price System (SCPS), representing a positive linear pricing rule:

Theorem 1.1 Let $(S, \kappa)$ be a pair of càdlàg adapted, locally bounded processes, with $\kappa$ nonnegative. Then (RNFLVR) $\Longleftrightarrow$ (SCPS).

(RNFLVR) is a robust version of the (NFLVR) condition of Delbaen \& Schachermayer (1994), and requires that (NFLVR) holds under an arbitrarily small perturbation of the bid-ask spread. Schachermayer (2004) shows that robustness arises naturally with transaction costs.

This paper formulates (RNFLVR) in terms of simple trading strategies. By contrast, in frictionless models (NFLVR) for simple strategies implies the semimartingale property for the asset price $S$ (Delbaen \& Schachermayer 1994, Theorem 7.1). Then, for any predictable strategy $H$ the gain process $(H \cdot S)$ becomes a well-defined stochastic integral, and the (NFLVR) for predictable strategies implies the existence of an equivalent local martingale measure.

Intuitively, this major difference arises from the impact of trading on wealth dynamics. In a frictionless market, only price changes affect wealth, while trading merely regulates their size. The result is a wide flexibility in trading patterns, represented by the large class of predictable strategies. By contrast, transaction costs entail a direct negative impact of trading on wealth, thereby restricting the class of feasible strategies.

The departure point of this paper is the concept of simple strategy, an almost surely finite sequence of left transactions, occurring immediately before a stopping time, and right transactions, occurring immediately afterwards (Definition 3.5). Rásonyi (2003) discovered that right-continuous strategies are not sufficient if prices may jump. Campi \& Schachermayer (2006) employed both types of transactions to prove a superreplication theorem.

A simple strategy is $x$-admissible if its future liquidation value (Definition 4.4) is greater than $-x$. This definition embodies the idea that the current value of a position 
should reflect sure future improvements in liquidity, and it reduces to the usual definition in frictionless arbitrage-free markets. From the technical viewpoint, this notion ensures that an admissible strategy with positive terminal value retains a positive value at all times (Proposition 4.9). This property is in turn crucial for the closedness of the set of admissible payoffs, which enables classic separation arguments. A Free Lunch with Vanishing Risk (Definition 5.2) is a sequence of simple admissible strategies which uniformly approximates an arbitrage payoff, and the (RNFLVR) property requires that no such sequences exist up to small model misspecifications.

Central to wealth dynamics is the concept of cost process, which tracks the cumulative cash flows generated by trading. In frictionless markets, the cost process $\int S d \theta$ is linked to the familiar gain process $\int \theta d S$ by the integration by parts formula:

$$
\int_{[0, T]} S_{t} d \theta_{t}=S_{T} \theta_{T}-S_{0} \theta_{0}-\int_{[0, T]} \theta_{t} d S_{t}
$$

which holds for a left-continuous, finite-variation strategy $\theta$ and a semimartingale price $S$. The left-hand side is a usual Stieltjes integral, while the right-hand side is as a stochastic integral, or, in the language of Dellacherie \& Meyer (1982), an elementary stochastic integral.

In the presence of bid and ask prices, the cost is the basic concept, since it avoids the appraisal of the risky position, which may be liquidated in the future at more favorable terms. The definition of cost is elementary for simple strategies (Definition 3.5), but its consistent extension to general (làdlàg) finite-variation strategies requires a careful study, which seems to be of independent interest and is developed in the appendix. From the mathematical standpoint, it is clear that the class of left-continuous strategies lacks any closedness property, hence it is unfit as domain of the integral operator. The economic intuition is also straightforward. When asset prices move in response to unpredictable (i.e. totally inaccessible) events, such as earthquakes, trading takes place only immediately after the event, i.e. the strategy $\theta$ is left-continuous. However, if market-sensitive information is announced at a predictable time $\tau$, as for quarterly earnings announcements and monetary policy meetings, it is perfectly feasible to trade both immediately before and immediately after the announcement. Then all the values $\theta_{\tau^{-}}, \theta_{\tau}, \theta_{\tau^{+}}$can be distinct, and yet economically relevant.

Building on this intuition, the appendix provides a consistent extension of the Stieltjes integral for a càdlàg integrand $S$, and a predictable finite variation integrator $\theta$. This integral is compatible with the usual stochastic integral: in the special case where $S$ is a semimartingale, the integration by parts formula (1.1) continues to hold for any predictable, finite-variation $\theta$, when the left-hand side is understood in the sense defined in the Appendix.

The rest of this paper is organized as follows: after a brief literature review, section 3 introduces the main model. The next section discusses the new concept of admissibility, and compares it to the frictionless case. The main result of this section is Proposition 4.9, which characterizes admissible strategies by their terminal payoffs. Section 5 introduces the (RNFLVR) condition, and establishes the Fatou closedness of the payoff space. Section 6 proves the main theorem using standard arguments: the Kreps-Yan theorem to separate the payoff space, and the argument of Jouini \& Kallal (1995) to obtain a martingale. The Appendix, which is readable without reference to 
the main text, constructs the predictable Stieltjes integral. The pathwise definition of the integral is probability free, and only requires a measurable space. The probability structure is needed for the construction of simple approximations.

\section{Literature Review}

The simple notion of arbitrage, as a positive nonzero payoff with zero price, proved effective in finite discrete time, leading to the FTAP of Dalang, Morton \& Willinger (1990), but becomes problematic in models with infinite horizons or continuous time. The seminal papers of Harrison \& Kreps (1979) and Kreps (1981) derived the existence of pricing rules assuming a stronger condition, the absence of Free Lunches, defined as nets, or generalized sequences, of payoffs converging to an arbitrage in a weak sense. Harrison \& Pliska (1981) highlighted the need for restrictions on trading strategies, to avoid arbitrage through doubling schemes.

The FTAP in continuous time for general càdlàg processes was established by Delbaen \& Schachermayer $(1994,1998)$, featuring the prominent rôle of semimartingale theory in Mathematical Finance. The notion of a Free Lunch with Vanishing Risk, a very strong approximation to an arbitrage payoff, proved key to establish first the semimartingale property of asset prices, then the existence of equivalent local martingale measures (in general, $\sigma$-martingale measures), formally equivalent to positive linear pricing rules.

Arbitrage theory with transaction costs was pioneered by Jouini \& Kallal (1995) in a continuous time model. They proved that the absence of free lunches is equivalent to the existence of a "shadow price", evolving within the bid-ask spread, and admitting an equivalent martingale measure. Subsequent work of Kabanov \& Stricker (2001a), Kabanov, Rásonyi \& Stricker (2002), and Schachermayer (2004) proved discrete time versions of the FTAP in increasing degrees of generality, in a numérairefree framework where all assets are freely exchangeable. Guasoni, Rásonyi \& Schachermayer (2010) characterize the absence of arbitrage with arbitrarily small transaction costs for continuous processes.

\section{The Model}

Consider a market model with one risky and one risk-free asset, based on a filtered probability space $\left(\Omega, \mathscr{F},\left(\mathscr{F}_{t}\right)_{t \in[0, T]}, P\right)$ satisfying the usual conditions, and set for convenience $\mathscr{F}_{t}:=\mathscr{F}_{T}$ for $t \geq T$. The risk-free asset is used as numéraire, hence its price is constantly equal to one. $S_{t}-\kappa_{t}$ and $S_{t}+\kappa_{t}$ denote respectively the bid (selling) and ask (buying) prices of the risky asset. Equivalently, each share trades at price $S_{t}$, and incurs a transaction fee of $\kappa_{t}$. The price-spread pair $(S, \kappa)$ satisfies the following:

Assumption 3.1 $(S, \kappa)$ is a pair of càdlàg adapted, locally bounded processes, and $\kappa$ is nonnegative.

Note that $S$ may become negative in this model. A nonnegative $\kappa$ is necessary to rule out static arbitrage by crossing the bid and ask prices. 
Remark 3.2 Alternatively, let $\underline{S} \leq \bar{S}$ be the bid and ask price processes. The relations $S_{t}:=\left(\underline{S}_{t}+\bar{S}_{t}\right) / 2$ and $\kappa_{t}=\bar{S}_{t}-S_{t}=S_{t}-\underline{S}_{t}$ link the bid-ask notation $(\underline{S}, \bar{S})$ to the pricespread notation $(S, \kappa)$. Thus, the choice of either notation does not restrict generality.

An investor trades in the risky asset according to the strategy $\left(\theta_{t}\right)_{t \in[0, T]}$, which represents the number of shares held at time $t$.

Definition 3.3 Let $\left(\sigma_{n}\right)_{n \geq 1}$ be a strictly increasing sequence of stopping times, such that $\sup _{n \geq 1} \sigma_{n}>T$ a.s., that is $P\left(\cup_{n \geq 1}\left\{\sigma_{n}>T\right\}\right)=1$. A simple strategy is a predictable process $\theta$ such that $\theta_{0}=\theta_{T}=0$ and:

$$
\theta=\sum_{n=1}^{\infty}\left(\theta_{\sigma_{n}} 1_{\llbracket \sigma_{n} \rrbracket}+\theta_{\sigma_{n}^{+}} 1_{\rrbracket \sigma_{n}, \sigma_{n+1} \llbracket}\right)
$$

For convenience, set $\theta_{s}=0$ for $s>T$.

According to this definition, a simple strategy entails a finite number of transactions, but this number may depend on $\omega \in \Omega$. Each stopping time $\sigma_{n}$ involves in principle two transactions: the left transaction from $\theta_{\sigma_{n}^{-}}$to $\theta_{\sigma_{n}}$ takes place at price $(S \pm \kappa)_{\sigma^{-}}$, just before a possible jump. The right transaction from $\theta_{\sigma_{n}}$ to $\theta_{\sigma_{n}^{+}}$takes place at price $(S \pm \kappa)_{\sigma}$, right after the jump.

Remark 3.4 Because $\theta$ is predictable, $\theta_{\sigma}=\theta_{\sigma^{-}}$for any totally inaccessible stopping time. Thus, left transactions may only take place on the accessible part of $\sigma$, while right transactions have no restrictions. This observation leads to an equivalent definition of simple strategy. Let $\left(\pi_{n}\right)_{n \geq 1}$ and $\left(\tau_{n}\right)_{n \geq 1}$ be two strictly increasing sequences of predictable times and stopping times respectively, such that $\sup _{n \geq 1} \pi_{n}>T$ and $\sup _{n \geq 1} \tau_{n}>T$ a.s. A simple strategy is a predictable process $\theta$ such that $\theta_{0}=\theta_{T}=0$ and (recalling the notation $\Delta \theta_{t}=\theta_{t}-\theta_{t^{-}}$and $\Delta^{+} \theta_{t}=\theta_{t^{+}}-\theta_{t}$ ):

$$
\theta=\sum_{n \geq 1} \Delta \theta_{\pi_{n}} 1_{\llbracket \pi_{n}, \infty \llbracket}+\sum_{n \geq 1} \Delta^{+} \theta_{\tau_{n}} 1_{\rrbracket} \tau_{n}, \infty \llbracket
$$

The equivalence with the original definition is straightforward: (3.2) implies (3.1) with $\left(\sigma_{n}\right)_{n \geq 1}$ as the ordered sequence obtained from $\left(\pi_{n}\right)_{n \geq 1}$ and $\left(\tau_{n}\right)_{n \geq 1}$. Viceversa, to obtain (3.2) from (3.1), set $\tau_{n}=\sigma_{n}$, and choose the sequence $\left(\pi_{n}\right)_{n>1}$ as the ordered sequence obtained from an exhausting sequence of the accessible parts of $\sigma_{n}$ (such ordered sequence exists because $\sigma_{n}$ is strictly increasing).

The definition of a simple strategy leads to the notion of cost:

Definition 3.5 The cost of a simple strategy $\theta$ is the random variable

$C(\theta)=\sum_{n=1}^{\infty}\left(S_{\sigma_{n}^{-}}\left(\theta_{\sigma_{n}}-\theta_{\sigma_{n-1}^{+}}\right)+S_{\sigma_{n}}\left(\theta_{\sigma_{n}^{+}}-\theta_{\sigma_{n}}\right)+\kappa_{\sigma_{n}^{-}}\left|\theta_{\sigma_{n}}-\theta_{\sigma_{n-1}^{+}}\right|+\kappa_{\sigma_{n}}\left|\theta_{\sigma_{n}^{+}}-\theta_{\sigma_{n}}\right|\right)$,

with the convention $\sigma_{0}^{+}=0$. Define the liquidation value as $V(\theta)=-C(\theta)$. The notation $V^{(S, \kappa)}(\theta)$ is used when ambiguity may arise. 
The first two terms in $C(\theta)$ represent the cash flows generated by trading at price $S$, which are positive for buying and negative for selling. The last two terms represent the transaction costs, which are always positive. Equivalently, $C(\theta)$ reflects purchases and sales respectively at bid and ask prices. To see this, regroup the terms in (3.3) as:

$$
\begin{aligned}
C(\theta) & =\sum_{n=1}^{\infty}\left[(S+\kappa)_{\sigma_{n}^{-}}\left(\theta_{\sigma_{n}}-\theta_{\sigma_{n-1}^{+}}\right)^{+}+(S+\kappa)_{\sigma_{n}}\left(\theta_{\sigma_{n}^{+}}-\theta_{\sigma_{n}}\right)^{+}\right] \\
& -\sum_{n=1}^{\infty}\left[(S-\kappa)_{\sigma_{n}^{-}}\left(\theta_{\sigma_{n}}-\theta_{\sigma_{n-1}^{+}}\right)^{-}+(S-\kappa)_{\sigma_{n}}\left(\theta_{\sigma_{n}^{+}}-\theta_{\sigma_{n}}\right)^{-}\right]
\end{aligned}
$$

Since a strategy begins and ends with cash only, the liquidation value is $-C(\theta)$.

\section{Admissibility}

To introduce admissibility, it is convenient to recast the notion of simple strategy in discrete-time notation, with the clock ticking at each transaction. This device overcomes the cumbersome distinction between left and right transactions.

Let $\theta$ be a simple strategy, and $\left(\sigma_{n}\right)_{n>1}$ the sequence of transaction times in Definition 3.3. Define the discrete filtration $(\hat{\mathscr{F}})_{n \geq 0}$ as $\hat{\mathscr{F}}=\left(\mathscr{F}_{0}, \mathscr{F}_{\sigma_{1}^{-}}, \mathscr{F}_{\sigma_{1}}, \mathscr{F}_{\sigma_{2}^{-}}, \mathscr{F}_{\sigma_{2}}, \ldots\right)$, that is $\hat{\mathscr{F}}_{2 n-1}=\mathscr{F}_{\sigma_{n}^{-}}$and $\hat{\mathscr{F}}_{2 n}=\mathscr{F}_{\sigma_{n}}$ for $n \geq 1$. The corresponding prices $(\hat{S}, \hat{\kappa})_{n \geq 0}$, defined analogously, are adapted to $\hat{\mathscr{F}}$ because $(S, \kappa)$ are adapted to $\mathscr{F}$. Using this notation, any simple strategy induces a $\hat{\mathscr{F}}$-adapted process $\left(\hat{\theta}_{n}\right)_{n \geq 0}$ defined as $\hat{\theta}=$ $\left(0, \theta_{\sigma_{1}}, \theta_{\sigma_{1}^{+}}, \theta_{\sigma_{2}}, \theta_{\sigma_{2}^{+}}, \ldots\right)$, or $\hat{\theta}_{2 n-1}=\theta_{\sigma_{n}}$ and $\hat{\theta}_{2 n}=\theta_{\sigma_{n}^{+}}$for $n \geq 1$.

$\hat{\mathscr{F}}_{n}$ represents the information available at the $n$-th transaction, and $\left(\hat{S}_{n}, \hat{\kappa}_{n}\right)$ the prices at which such a transaction takes place. The "hat operator" maps predictable to adapted processes, in the following sense:

Proposition 4.1 Let $\tau_{n}=\sigma_{n A_{n}}:=\sigma_{n} 1_{A_{n}}+\infty 1_{\Omega \backslash A_{n}}$ denote the totally inaccessible part of $\sigma_{n}$, for some $A_{n} \in \mathscr{F}_{\sigma_{n}}=\hat{\mathscr{F}}_{2 n}$.

i) If $\left(\theta_{t}\right)_{t \geq 0}$ is a simple strategy, then $\left(\hat{\theta}_{n}\right)_{n \geq 0}$ is $\hat{\mathscr{F}}$-adapted. In addition, $\hat{\theta}_{2 n-1}=$ $\theta_{\sigma_{n}}=\theta_{\sigma_{n}^{-}}=\hat{\theta}_{2(n-1)}$ on $A_{n}$.

ii) If $\left(\eta_{n}\right)_{n \geq 0}$ is $\hat{\mathscr{F}}$-adapted, and $\eta_{2 n-1}=\eta_{2(n-1)}$ on $A_{n}$, then $\theta=\sum_{n \geq 0}\left(\Delta \eta_{2 n-1} 1_{\llbracket \sigma_{n}, \infty \llbracket}+\right.$ $\left.\Delta \eta_{2 n} 1_{\rrbracket \sigma_{n}, \infty \llbracket}\right)$ is a simple strategy, and $\hat{\theta}=\eta$ (recall that $\Delta \eta_{n}=\eta_{n}-\eta_{n-1}$ ).

Proof For $i$ ), observe that $\theta_{\sigma}$ is $\mathscr{F}_{\sigma^{-}}$-measurable because $\theta$ is predictable, and that $\theta_{\sigma^{+}}$is $\mathscr{F}_{\sigma^{-}}$-measurable since the filtration is right-continuous. Since $\sigma_{n A_{n}}$ is totally inaccessible, it follows that $\theta_{\sigma_{n}}=\theta_{\sigma_{n}-}$ on $A_{n}$.

To obtain $i i$ ), it suffices to show that both terms in the definition of $\theta$ are pre-

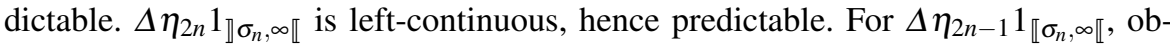
serve that $\Delta \eta_{2 n-1}=0$ on $A_{n}$ by assumption. Since $\sigma_{n \Omega \backslash A_{n}}$ is the accessible part of $\sigma_{n}$, its graph admits the representation:

$$
\llbracket \sigma_{n \Omega \backslash A_{n}} \rrbracket=\cup_{m \geq 1} \llbracket \pi_{m} \rrbracket
$$


where $\left(\pi_{m}\right)_{m \geq 1}$ is a sequence of predictable times, whose graphs $\left(\llbracket \pi_{m} \rrbracket\right)_{m \geq 1}$ are pairwise disjoint. Thus, $\Delta \eta_{2 n-1} 1_{\llbracket \pi_{m}, \infty \llbracket}$ is predictable for each $m$ (Jacod \& Shiryaev 2003, 1.2.12), hence also $\Delta \eta_{2 n-1} 1_{\llbracket \sigma_{n}, \infty \llbracket}=\Delta \eta_{2 n-1} \sum_{m \geq 1} 1_{\llbracket \pi_{m}, \infty \llbracket}$ is predictable.

Definition 4.2 A random partition is an increasing sequence of stopping times $\Sigma=$ $\left(\sigma_{n}\right)_{n \geq 1}$, such that $\sup _{n \geq 1} \sigma_{n}>T$ a.s. (i.e. $P\left(\cup_{n \geq 1}\left\{\sigma_{n} \wedge T=T\right\}\right)=1$ ), and $P\left(\sigma_{n} \neq\right.$ $\left.\sigma_{n-1}\right)>0$ for all $n$ (i.e., any stopping time appears only once). If a stopping time appears at most twice, that is $\sigma_{n}=\sigma_{n-1}$ a.s. implies that $P\left(\sigma_{n} \neq \sigma_{n+1}\right)>0$, then $\Sigma$ is an extended partition.

A random or extended partition $\Sigma^{\prime}$ is finer than $\Sigma$ if $\cup_{n \geq 1} \llbracket \sigma_{n} \rrbracket \subset \cup_{n \geq 1} \llbracket \sigma_{n}^{\prime} \rrbracket$, that is $\cup_{n \geq 1} \llbracket \sigma_{n} \rrbracket \backslash \cup_{n \geq 1} \llbracket \sigma_{n}^{\prime} \rrbracket$ is evanescent. For a random or extended partition $\Sigma, \widehat{\Sigma}$ denotes the corresponding partition in discrete time notation.

Remark 4.3 The unusual choice to allow a stopping time $\sigma_{n}$ to appear twice in a partition allows to use the same stopping time both for freezing, and for closing, two concepts to be introduced shortly.

Define the cumulative cost process $\left(\hat{C}_{k}(\theta)\right)_{k \geq 0}$ as:

$$
\hat{C}_{k}(\hat{\theta})=\sum_{n=1}^{k}\left(\hat{S}_{n}\left(\hat{\theta}_{n}-\hat{\theta}_{n-1}\right)+\hat{\kappa}_{n}\left|\hat{\theta}_{n}-\hat{\theta}_{n-1}\right|\right)
$$

which implies that $V(\theta)=-C(\theta)=-\hat{C}_{\infty}(\hat{\theta})$. By definition, $\hat{V}(\hat{\theta}):=V(\theta)$.

This notation streamlines the definition of an admissible strategy by avoiding the distinction between left and right transactions. Intuitively, a strategy is $x$-admissible if, after any transaction, the clearing broker can freeze the agent's account, and liquidate it to cash at a later date for a cost less than $x$. This condition ensures that a credit line of $x$ is effectively in force.

Definition 4.4 For $x \geq 0$, a simple strategy $\theta$ is $x$-admissible if, for any random partition $\Sigma$ defining $\theta$, there exists some finer extended partition $\Sigma^{\prime} \supseteq \Sigma:=\left\{\sigma_{n}, n \in \mathbb{N}\right\}$, for all $k \geq 0$ with $k \in \widehat{\Sigma}^{\prime} \cap \widehat{\Sigma}$, there exists another simple strategy ${ }^{k} \theta$, called liquidation strategy, such that:

i) $\widehat{{ }^{k} \theta}:={ }^{k} \hat{\theta}:=\hat{\theta}_{\cdot \wedge k} 1_{\left\{\cdot<\lambda_{k}\right\}}$ for some $\hat{\mathscr{F}}$-stopping time $\lambda_{k}>k$ a.s. with $\lambda_{k} \in \widehat{\Sigma^{\prime}}$, the liquidation time.

ii) $x+V\left({ }^{k} \theta\right) \geq 0$.

$\mathscr{A}_{x}^{s}$ denotes the set of simple $x$-admissible strategies, and $\mathscr{A}^{s}=\cup_{x>0} \mathscr{A}_{x}^{s}$ the set of simple admissible strategies. A simple arbitrage is $\theta \in \mathscr{A}^{s}$ such that $P(V(\theta) \geq 0)=1$ and $P(V(\theta)>0)>0$. A market satisfies (NA-S) if $\theta \in \mathscr{A}^{s}$ and $P(V(\theta) \geq 0)=1$ implies that $V(\theta)=0$.

Remark 4.5 Equivalently, Definition 4.4 defines an admissible strategy as a simple strategy that can be frozen at any stopping time. Also, the statements of Definition 4.4 remain valid for any extended partition finer than $\Sigma^{\prime}$, and the extended partition does not change the value of the strategy. 
Remark 4.6 In Definition 4.4, the freeze occurs after a fixed transaction, but it could equivalently occur at any $\hat{\mathscr{F}}$-stopping time $\sigma$. Indeed, if $\left(\lambda_{k}\right)_{k \geq 1}$ are the liquidation times in Definition 4.4, then $\lambda_{\sigma}=\sum_{k=1}^{\infty} \lambda_{k} 1_{\{\sigma=k\}}$ is a liquidation time for $\sigma$.

Remark 4.7 The liquidation strategy ${ }^{k} \hat{\theta}$ satisfies ${ }^{k} \hat{\theta}_{n}=\hat{\theta}_{n} 1_{\{n<k\}}+\hat{\theta}_{k} 1_{\left\{k \leq n<\lambda_{k}\right\}}$, and induces a unique $\mathscr{F}$-predictable process ${ }^{k} \theta$, which satisfies ${ }^{k} \boldsymbol{\theta}={ }^{k} \hat{\boldsymbol{\theta}}$ and is piecewise constant between $\sigma_{k}$ and $\sigma_{k+1} \cdot{ }^{k} \theta$ takes six possible forms, depending on whether the freeze $(k)$ and liquidation $\left(\lambda_{k}\right)$ are left or right transactions. With $k=2 n_{k}-1$ or $k=2 n_{k}$ and $\lambda_{k}=2 \widetilde{n}_{k}-1$ or $\lambda_{k}=2 \widetilde{n}_{k}$

$$
\begin{aligned}
& \theta 1_{\llbracket 0, \sigma_{n_{k}} \rrbracket}+\theta_{\sigma_{n_{k}}^{+}} 1_{\rrbracket \sigma_{n_{k}}, \sigma_{\tilde{n}_{k}} \rrbracket} \\
& \theta 1_{\llbracket 0, \sigma_{n_{k}} \rrbracket}+\theta_{\sigma_{n_{k}}^{+}} 1_{\rrbracket \sigma_{n_{k}}, \sigma_{\tilde{n}_{k}} \llbracket} \\
& \theta 1_{\llbracket 0, \sigma_{n_{k}} \llbracket}+\theta_{\sigma_{n_{k}}} 1_{\llbracket \sigma_{n_{k}}, \sigma_{\tilde{n}_{k}} \rrbracket} \\
& \theta 1_{\llbracket 0, \sigma_{n_{k}} \llbracket}+\theta_{\sigma_{n_{k}}} 1_{\llbracket \sigma_{n_{k}}, \sigma_{\tilde{n}_{k}} \llbracket}
\end{aligned}
$$

If $\lambda_{k}=k+1$, then $\sigma_{n_{k}}$ may coincide with $\sigma_{\tilde{n}_{k}}$, which amounts to freezing $\theta_{\sigma_{n_{k}}^{+}}$(respectively $\theta_{\sigma_{n_{k}}}$ ), and liquidate immediately the position, without waiting another tick of the clock $\Sigma$.

Compare definition (4.4) to its frictionless counterpart:

$$
x+V\left(\theta 1_{[0, t]}\right) \geq 0 \quad \text { for all } t \in[0, T]
$$

In general, (4.3) is more restrictive, but in a frictionless, arbitrage free market, the two definitions are equivalent:

Proposition 4.8 Let $\kappa=0$. For any $\theta \in \mathscr{A}_{x}^{s}$, either:

$$
\sum_{n=1}^{k} \hat{\theta}_{n-1}\left(\hat{S}_{n}-\hat{S}_{n-1}\right) \geq-x \quad \text { a.s. for all } k \geq 0
$$

or there exists an arbitrage opportunity.

Proof If $\kappa=0$, observe that:

$$
V(\theta)=-\sum_{n=1}^{\infty} \hat{S}_{n}\left(\hat{\theta}_{n}-\hat{\theta}_{n-1}\right)=\sum_{n=1}^{\infty} \hat{\theta}_{n-1}\left(\hat{S}_{n}-\hat{S}_{n-1}\right)
$$

and therefore:

$$
\begin{aligned}
\hat{V}\left(\hat{\theta} 1_{\{\cdot<k\}}\right) & =\sum_{n=1}^{k} \hat{\theta}_{n-1}\left(\hat{S}_{n}-\hat{S}_{n-1}\right) \\
V\left({ }^{k} \theta\right) & =\left(\sum_{n=1}^{k} \hat{\theta}_{n-1}\left(\hat{S}_{n}-\hat{S}_{n-1}\right)\right)+\hat{\theta}_{k}\left(\hat{S}_{\lambda_{k}}-\hat{S}_{k}\right)
\end{aligned}
$$

If $\lambda_{k-1}=k$ is not a liquidation time, there exists $A \in \hat{\mathscr{F}}_{k}$ on which $V\left(\theta 1_{\{<<k\}}\right)<-x$. Thus, the buy-and-hold strategy $\hat{\eta}_{n}=\hat{\theta}_{k} 1_{A \cap\left\{k \leq n<\lambda_{k}\right\}}$ is an arbitrage opportunity. 
The main result of this section characterizes an $x$-admissible strategy by its terminal payoff. This property essentially confirms that Definition 4.4 is the relevant notion of admissibility in this model.

Proposition 4.9 If (NA-S) holds, then $\mathscr{A}_{x}^{s}=\left\{\theta \in \mathscr{A}^{s}: x+V(\theta) \geq 0\right.$ a.s. $\}$.

The nontrivial statement is that $\theta \in \mathscr{A}_{x}^{s}$ if $\theta \in \mathscr{A}^{s}$ and $x+V(\theta) \geq 0$ a.s. In a frictionless market, this is a straightforward consequence of absence of arbitrage and the concatenation property $V(\theta)=V\left(\theta 1_{[0, t]}\right)+V\left(\theta 1_{(t, T]}\right)$. However, concatenation fails under transaction costs, since splitting a strategy at time $t$ creates two new transactions, thereby increasing costs.

The next proof exploits the following idea: if a strategy has positive final liquidation value, then after every transaction there is either a liquidation time, or an arbitrage. The trouble is that the arbitrage is not necessarily the remaining part of the strategy (because concatenation fails), but it may be hidden inside. Discovering the arbitrage involves the elimination of "damaging transactions", the ones executed at a worse (either bid or ask) price than the average liquidation price required by the current position. This elimination process eventually leads to either a liquidation time, or an arbitrage.

Proof The inclusion $\mathscr{A}_{x}^{s} \subset\left\{\theta \in \mathscr{A}^{s}: x+V(\theta) \geq 0\right.$ a.s. $\}$ is straightforward: if $\theta \in$ $\mathscr{A}_{x}^{s}$, by assumption the strategies $\left({ }^{k} \hat{\theta}\right)_{k>0}$ satisfy $x+V\left({ }^{k} \theta\right) \geq 0$ for all $k \geq 0$. Since for a.e. $\omega,{ }^{k} \hat{\theta}_{n}(\omega)=\hat{\theta}_{n}(\omega)$ for all $n$ and for $k \geq k_{0}(\omega)$ large enough, it follows that $x+V(\theta) \geq 0$ a.s.

For the reverse inclusion, the following argument constructs explicitly a liquidation time $\lambda_{k} \in \widehat{\Sigma}^{\prime}$ from the initial assumption that $x+V(\theta)=x-\hat{C}_{\infty}(\hat{\theta}) \geq 0$ and $\theta \in \mathscr{A}^{s}$. Since $\theta \in \mathscr{A}_{y}^{s}$ for some $y>0$, consider the finer extended partition $\Sigma^{\prime} \supseteq \Sigma:=\left\{\sigma_{n}: n \in \mathbb{N}\right\}$ as in Definition 4.4 , where $\Sigma$ defines the strategy $\theta$. The proof proceeds in a recursive, "algorithmic" fashion, using $m$ as counter, initially equal to 0 .

Step 1: Find arbitrage or liquidation.

After the $k$-th transaction, the portfolio consists of $\hat{\theta}_{k}$ shares and a cash position equal to $-\hat{C}_{k}(\hat{\theta})$. Consider the following $\hat{\mathscr{F}}_{k+1}$-partition of $\Omega$ :

$$
\begin{array}{lr}
A_{+}=\left\{\hat{\theta}_{k}>0, \hat{\theta}_{k+1}>0\right\} & \text { (stay long) } \\
A_{-}=\left\{\hat{\theta}_{k}<0, \hat{\theta}_{k+1}<0\right\} & \text { (stay short) } \\
A_{ \pm}=\left\{\hat{\theta}_{k+1} \hat{\theta}_{k} \leq 0\right\} & \text { (long/short change) }
\end{array}
$$

and define $\hat{\eta}=\hat{\theta} 1_{\{: \leq k\}},\left(\hat{\eta}_{n}=\hat{\theta}_{n}\right.$ for $n \leq k$ and $\hat{\eta}_{n}=0$ for $\left.n \geq k+1\right)$, and $\hat{\zeta}=$ $\hat{\theta} 1_{\{. \geq k+1\}}=\hat{\theta}-\hat{\eta}$. We claim that $x+V(\eta) \geq 0$ a.s. on $A_{ \pm}$, otherwise $\hat{\zeta}$ is an arbitrage on the event $G:=\{x+V(\eta)<0\}$. To see this, note that $\left|\hat{\theta}_{k}-\hat{\theta}_{k+1}\right|=\left|\hat{\theta}_{k}\right|+\left|\hat{\theta}_{k+1}\right|$ on $A_{ \pm}$, and therefore $\hat{C}(\hat{\theta})=\hat{C}(\hat{\eta})+\hat{C}(\hat{\zeta})$. Since $\theta \in \mathscr{A}_{y}^{s}$, for any $j \geq k$ there is some liquidation time $\lambda_{j} \in \widehat{\Sigma}^{\prime}$ for $\hat{\theta}$. Then, on $G$ the following relations hold:

$$
\begin{aligned}
V\left(\hat{\zeta}_{\cdot \wedge j} 1_{\left\{\cdot<\lambda_{j}\right\}}\right) & =-C\left(\hat{\theta}_{\cdot \wedge j} 1_{\left\{\cdot<\lambda_{j}\right\}}\right)+C(\hat{\eta}) \geq x-y \\
V(\hat{\zeta}) & =(x+V(\hat{\theta}))-(x+V(\hat{\eta}))>0
\end{aligned}
$$


The first inequality proves that $\hat{\zeta} 1_{G} \in \mathscr{A}_{y-x}^{s}$, and the second one that it is an arbitrage on $G$. Thus, $\lambda_{k}=k+1$ is a liquidation time on $A_{ \pm}$.

On $A_{+}$("stay long"), denote by $l=\frac{\hat{C}_{k}(\hat{\theta})-x}{\hat{\theta}_{k}}$ the minimum average liquidation price for solvability. Distinguish now three cases:

$$
\begin{array}{ll}
A_{+}^{l}=A_{+} \cap\left\{\hat{\theta}_{k+1}-\hat{\theta}_{k}>0,(\hat{S}+\hat{\kappa})_{k+1}<l\right\} & \text { (buy low) } \\
A_{+}^{h}=A_{+} \cap\left\{\hat{\theta}_{k+1}-\hat{\theta}_{k}<0,(\hat{S}-\hat{\kappa})_{k+1}>l\right\} & \text { (sell high) } \\
A_{+}^{s}=A_{+} \backslash\left(A_{+}^{h} \cup A_{-}^{l}\right) &
\end{array}
$$

First, observe that on $A_{+}^{h}$ ("sell high") $\lambda_{k}=k+1$ is a liquidation time by definition. Second, on $A_{+}^{l}$ ("buy low"), an arbitrage opportunity arises, hence $P\left(A_{+}^{l}\right)=0$. This is intuitively clear, as the knowledge that the current long position $\hat{\theta}_{k}$ will be sold at least at price $l$ guarantees that any purchase below $l$ price yields an arbitrage. To see this formally, set $\hat{\zeta}=\hat{\theta} 1_{\{\cdot \geq k+1\}}$ as before. To check that $\hat{\zeta}$ is admissible, note that, since $\theta \in \mathscr{A}_{y}^{s}$, it has a liquidation time $\lambda_{j} \in \widehat{\Sigma}^{\prime}$ for all $j \geq k+1$. On $A_{+}^{l}$ the value of $\hat{\zeta}_{\cdot \wedge j} 1_{\left\{\cdot<\lambda_{j}\right\}}$ is equal to:

$$
V\left(\hat{\zeta}_{\cdot \wedge j} 1_{\left\{\cdot<\lambda_{j}\right\}}\right)=V\left(\hat{\theta}_{\cdot \wedge j} 1_{\left\{\cdot<\lambda_{j}\right\}}\right)+\hat{C}_{k}(\hat{\theta})-(\hat{S}+\hat{\kappa})_{k+1} \hat{\theta}_{k}
$$

Note the term $(\hat{S}+\hat{\kappa})_{k+1} \hat{\theta}_{k}$ in the right-hand side, which reflects the additional purchase of $\hat{\theta}_{k}$ shares, necessary to build a position of $\hat{\theta}_{k+1}$ shares from 0 at the $k+1$ transaction. Since $y+V\left(\hat{\theta}_{\cdot \wedge j} 1_{\left\{\cdot<\lambda_{j}\right\}}\right) \geq 0$ by assumption, on $A_{+}^{l}$ it follows that:

$$
\begin{aligned}
V\left(\hat{\zeta}_{\cdot \wedge j} 1_{\left\{\cdot<\lambda_{j}\right\}}\right) & =\left(y+V\left(\hat{\theta}_{\cdot \wedge j} 1_{\left\{\cdot<\lambda_{j}\right\}}\right)\right)-y+\hat{C}_{k}(\hat{\theta})-(\hat{S}+\hat{\kappa})_{k+1} \hat{\theta}_{k} \\
& \geq-y+\hat{C}_{k}(\hat{\theta})-(\hat{S}+\hat{\kappa})_{k+1} \hat{\theta}_{k} \\
& =-y+x+\hat{\theta}_{k}\left(l-(\hat{S}+\hat{\kappa})_{k+1}\right)>x-y
\end{aligned}
$$

which proves that $\zeta \in \mathscr{A}_{y-x}^{s}\left(\zeta 1_{A_{+}^{l}} \in \mathscr{A}_{y-x}^{s}\right)$. In addition, since $x+V(\theta) \geq 0$ by assumption, setting $y=x$ and $j=\infty$ in the above equation shows that $\hat{\zeta}$ is an arbitrage.

The situation for $A_{-}$("stay short") is symmetric, in that liquidation is trivial on the "buy low" case $A_{-}^{l}=A_{-} \cap\left\{\hat{\theta}_{k+1}-\hat{\theta}_{k}>0,(\hat{S}+\hat{\kappa})_{k+1}<l\right\}$, while arbitrage arises in the "sell high" case $A_{-}^{h}=A_{-} \cap\left\{\hat{\theta}_{k+1}-\hat{\theta}_{k}<0,(\hat{S}-\hat{\kappa})_{k+1}>l\right\}$. In summary, liquidation at the $(k+1)$-th transaction is feasible on the event $B_{m}=A_{ \pm} \cup A_{+}^{h} \cup A_{-}^{l}$ (the counter $m$ is used here).

Step 2: Else skip a damaging transaction.

It remains to consider the cases $A_{+}^{s}$ and $A_{-}^{s}$. Intuitively, any transaction taking place on these events is "damaging", since it neither buys low, nor it sells high. To skip this transaction, while ensuring positive final liquidation, rescale now the remaining part of the strategy. Formally, define $\hat{\zeta}$ as $\hat{\zeta}_{n}=\hat{\theta}_{n}$ for $n \leq k$, and $\hat{\zeta}_{n}=\frac{\hat{\theta}_{k}}{\hat{\theta}_{k+1}} \hat{\theta}_{n}$ for $n \geq k+1$. 
Then note that:

$$
\begin{aligned}
0 & \leq x+V(\theta) \\
& =x-\hat{C}_{k}(\hat{\theta})-(\hat{S} \pm \hat{\kappa})_{k+1}\left(\hat{\theta}_{k+1}-\hat{\theta}_{k}\right)-\left(\hat{C}_{\infty}(\hat{\theta})-\hat{C}_{k+1}(\hat{\theta})\right) \\
& \leq x-\hat{C}_{k}(\hat{\theta})+\frac{\hat{\theta}_{k+1}-\hat{\theta}_{k}}{\hat{\theta}_{k}}\left(x-\hat{C}_{k}(\hat{\theta})\right)-\left(\hat{C}_{\infty}(\hat{\theta})-\hat{C}_{k+1}(\hat{\theta})\right) \\
& =\frac{\hat{\theta}_{k+1}}{\hat{\theta}_{k}}\left(x-\hat{C}_{k}(\hat{\theta})-\frac{\hat{\theta}_{k}}{\hat{\theta}_{k+1}}\left(\hat{C}_{\infty}(\hat{\theta})-\hat{C}_{k+1}(\hat{\theta})\right)\right) \\
& =\frac{\hat{\theta}_{k+1}}{\hat{\theta}_{k}}(x+V(\zeta)),
\end{aligned}
$$

where the \pm in the second line equals + on $\left(A_{+}^{s} \cup A_{-}^{s}\right) \cap\left\{\hat{\theta}_{k+1}-\hat{\theta}_{k}>0\right\}$, and equals to - on $\left(A_{+}^{s} \cup A_{-}^{s}\right) \cap\left\{\hat{\theta}_{k+1}-\hat{\theta}_{k}<0\right\}$. Since the strategy $\hat{\zeta}$ satisfies the same assumptions as $\hat{\theta}$, and coincides with $\hat{\theta}$ up to (and including) the $k$-th transaction, but with $\hat{\zeta}_{k+m}=$ $\hat{\theta}_{k}, m=1$, a liquidation time for $\hat{\zeta}$ is also valid for $\hat{\theta}$. Thus, on the event $A_{+}^{s} \cup A_{-}^{s}$ replace $\hat{\theta}$ by $\hat{\zeta}$ and return to Step 1 , increasing $m$ by one.

Since the number of transactions in a simple strategy is a.s. finite, this recursion takes place only a finite number of times, i.e. $P\left(\cup_{m \geq 1} B_{m}\right)=1$. The liquidation time is thus $\lambda_{k}=\sum_{m=0}^{\infty}(k+1+m) 1_{B_{m} \backslash \cup_{j<m} B_{j}}$.

One last check is necessary: the strategy ${ }^{k} \hat{\theta}$ must induce a $\mathscr{F}$-predictable strategy ${ }^{k} \theta$. By Proposition 4.1, this is the case if ${ }^{k} \hat{\theta}$ is $\hat{F}$-adapted, and ${ }^{k} \hat{\theta}_{2 n-1}={ }^{k} \hat{\theta}_{2(n-1)}$ on $A_{n}$ (which defines the totally inaccessible part of $\sigma_{n}$ in Proposition 4.1). ${ }^{k} \hat{\theta}$ is $\hat{\mathscr{F}}$ adapted because $\theta$ is $\hat{\mathscr{F}}$-adapted and $\left\{\lambda_{k}=k+m\right\}$ is $\hat{\mathscr{F}}_{k+m}$-measurable (the events $A_{ \pm}, A_{+}^{h}, A_{-}^{l}$ in the construction depend only on $\hat{\theta}_{k+i}$ for $\left.i \leq m\right)$. For the predictability part, observe that the original strategy satisfies $\hat{\theta}_{2 n}=\hat{\theta}_{2 n-1}$ on $A_{n}$ by assumption. Thus it suffices to check that for $k=2 n-1$ the operations of liquidation and scaling in the above construction preserve this property. Indeed, the events $A_{+}^{h}, A_{-}^{l}$ are disjoint from $\left\{\hat{\theta}_{k+1}=\hat{\theta}_{k}\right\}$, hence liquidation (i.e. setting ${ }^{k} \hat{\theta}_{k+1}=0$ ) takes place outside $A_{n}$ Regarding $A_{ \pm}$, note that $A_{ \pm} \cap\left\{\hat{\theta}_{k+1}=\hat{\theta}_{k}\right\}=\left\{\hat{\theta}_{k+1}=\hat{\theta}_{k}=0\right\}$, and liquidation does not alter this property, since ${ }^{k} \hat{\theta}_{k+1}={ }^{k} \hat{\theta}_{k}=0$. Finally, the scaling operation $\hat{\zeta}_{n}=$ $\frac{\hat{\theta}_{k}}{\hat{\theta}_{k+1}} \hat{\theta}_{n}$ for $n \geq k+1$ entails that, on the event $\left\{\hat{\theta}_{k+1}=\hat{\theta}_{k}\right\}$, the property $\left\{\hat{\zeta}_{k+1}=\hat{\zeta}_{k}\right\}$ holds everywhere by definition of $\hat{\zeta}$.

A similar, but simpler, argument shows that the set of admissible strategies is convex. This fact is obvious in frictionless markets, but not in this setting.

Lemma 4.10 If (NA-S) holds, then $\mathscr{A}^{s}$ is convex.

Proof If $\theta \in \mathscr{A}_{x}^{s}$ and $c>0$, then $c \theta \in \mathscr{A}_{c x}^{s}$ because a liquidation time for $\theta$ is also a liquidation time for $c \theta$. Convexity follows by showing that if $\theta \in \mathscr{A}_{x}^{s}$ and $\eta \in \mathscr{A}_{y}^{s}$, then $\theta+\eta \in \mathscr{A}_{x+y}^{s}$. To this end, consider a common finer extended partition $\widehat{\Sigma}^{\prime}$ for which the properties of Definition 4.4 hold for both $\theta$ and $\eta$ defined by a common 
random partition $\Sigma$ containing the one defining $\theta+\eta$. Denote respectively by $\lambda_{k} \in \widehat{\Sigma^{\prime}}$ and $\mu_{k} \in \widehat{\Sigma}^{\prime}$ the liquidation times of $\theta$ and $\eta$ at time $k \in \widehat{\Sigma} \cap \widehat{\Sigma^{\prime}}$.

Let $\lambda_{k}$ and $\mu_{k}$ be the liquidation times of $\theta$ and $\eta$ respectively. Consider first the event $\left\{\hat{\theta}_{k}+\hat{\eta}_{k}>0\right\}$, and split it into four cases (excluding the trivial case $\lambda_{k}=\mu_{k}$ ):

$$
\begin{aligned}
& \left\{\hat{\theta}_{k}+\hat{\eta}_{k}>0, \lambda_{k}<\mu_{k}, \hat{\eta}_{k} \geq 0\right\} \\
& \left\{\hat{\theta}_{k}+\hat{\eta}_{k}>0, \lambda_{k}<\mu_{k}, \hat{\eta}_{k}<0\right\} \\
& \left\{\hat{\theta}_{k}+\hat{\eta}_{k}>0, \lambda_{k}>\mu_{k}, \hat{\eta}_{k} \geq 0\right\} \\
& \left\{\hat{\theta}_{k}+\hat{\eta}_{k}>0, \lambda_{k}>\mu_{k}, \hat{\eta}_{k}<0\right\}
\end{aligned}
$$

$\lambda_{k}$ liquidates $\theta+\eta$ in the second case, otherwise $\hat{\eta}_{k} 1_{\left\{\lambda_{k} \leq<<\mu_{k}\right\}}$ is an arbitrage. Similarly, $\mu_{k}$ liquidates $\theta+\eta$ in the fourth case, otherwise $\hat{\theta}_{k} 1_{\left\{\mu_{k} \leq-<\lambda_{k}\right\}}$ is an arbitrage. In the first and the third cases, on the even $\left\{\hat{\theta}_{k} \geq 0\right\}$ consider the candidate liquidation strategies $\hat{\zeta}^{\lambda}=(\hat{\theta}+\hat{\eta})_{\cdot \wedge k} 1_{\left\{\cdot<\lambda_{k}\right\}}$ and $\hat{\zeta}^{\mu}=(\hat{\theta}+\hat{\eta})_{\cdot \wedge k} 1_{\left\{\cdot<\mu_{k}\right\}}$, and note that:

$$
\begin{aligned}
V\left(\hat{\zeta}^{\lambda}\right) & =-\hat{C}_{k}(\hat{\theta}+\hat{\eta})+(S-\kappa)_{\lambda_{k}}\left(\hat{\theta}_{k}+\hat{\eta}_{k}\right) \\
V\left(\hat{\zeta}^{\mu}\right) & =-\hat{C}_{k}(\hat{\theta}+\hat{\eta})+(S-\kappa)_{\mu_{k}}\left(\hat{\theta}_{k}+\hat{\eta}_{k}\right) \\
V\left({ }^{k} \hat{\theta}+{ }^{k} \hat{\eta}\right) & =-\hat{C}_{k}(\hat{\theta}+\hat{\eta})+(S-\kappa)_{\lambda_{k}} \hat{\theta}_{k}+(S-\kappa)_{\mu_{k}} \hat{\eta}_{k} \\
& =\frac{\hat{\theta}_{k}}{\hat{\theta}_{k}+\hat{\eta}_{k}} V\left(\hat{\zeta}^{\lambda}\right)+\frac{\hat{\eta}_{k}}{\hat{\theta}_{k}+\hat{\eta}_{k}} V\left(\hat{\zeta}^{\mu}\right)
\end{aligned}
$$

Thus, in such cases $V\left({ }^{k} \hat{\theta}+{ }^{k} \hat{\eta}\right)$ is a convex combination of $V\left(\hat{\zeta}^{\lambda}\right)$ and $V\left(\hat{\zeta}^{\mu}\right)$. Since

$$
V\left({ }^{k} \hat{\boldsymbol{\theta}}+{ }^{k} \hat{\eta}\right) \geq V\left({ }^{k} \hat{\boldsymbol{\theta}}\right)+V\left({ }^{k} \hat{\eta}\right) \geq-(x+y)
$$

it follows that either $V\left(\hat{\zeta}^{\lambda}\right) \geq-(x+y)$ or $V\left(\hat{\zeta}^{\mu}\right) \geq-(x+y)$, otherwise (4.16) is violated. Thus, the liquidation time of $\theta+\eta$ is a mixture of liquidation times of $\theta$ and $\eta$. To finish the discussion on the event $\left\{\hat{\theta}_{k}+\hat{\eta}_{k}>0\right\}$, it remains to consider the first and third case with $\hat{\theta}_{k}<0$. Then:

$$
\begin{gathered}
V\left({ }^{k} \hat{\boldsymbol{\theta}}+{ }^{k} \hat{\eta}\right)=-\hat{C}_{k}(\hat{\boldsymbol{\theta}}+\hat{\eta})+(S+\kappa)_{\lambda_{k}} \hat{\theta}_{k}+(S-\kappa)_{\mu_{k}} \hat{\eta}_{k} \\
x+y-C_{k}(\hat{\theta}+\hat{\eta}) \geq x+y-C_{k}(\hat{\theta})-C_{k}(\hat{\eta}) \geq-\hat{\eta}_{k}(S-\kappa)_{\mu_{k}}-\hat{\theta}_{k}(S+\kappa)_{\lambda_{k}}
\end{gathered}
$$

from which it follows that:

$$
x+y-C_{k}(\hat{\theta}+\hat{\eta})+\left(\hat{\theta}_{k}+\hat{\eta}_{k}\right)(S-\kappa)_{\mu_{k}} \geq \hat{\theta}_{k}\left((S-\kappa)_{\mu_{k}}-(S+\kappa)_{\lambda_{k}}\right)
$$

This inequality implies that $\mu_{k}$ is a liquidation time, otherwise the right-hand side would be a buy-and-hold arbitrage.

The discussion on the event $\left\{\hat{\theta}_{k}+\hat{\eta}_{k}<0\right\}$ is symmetric, therefore it remains the case $\left\{\hat{\theta}_{k}+\hat{\eta}_{k}=0\right\}$. By symmetry, it is enough to consider $\left\{\hat{\boldsymbol{\theta}}_{k}+\hat{\eta}_{k}=0, \theta_{k} \geq 0\right\}$. By assumption, $x-C_{k}(\theta)+\hat{\theta}_{k}(\hat{S}-\hat{\kappa}) \lambda_{k} \geq 0$ and $-C_{k}(\eta)+\hat{\eta}_{k}(\hat{S}+\hat{\kappa})_{\mu_{k}} \geq 0$. Hence:

$$
x+y-C_{k}(\theta)-C_{k}(\eta) \geq-\hat{\theta}_{k}\left((\hat{S}-\hat{\kappa}) \lambda_{k}-(\hat{S}+\hat{\kappa})_{\mu_{k}}\right)
$$

This inequality, combined with $V\left({ }^{k}(\theta+\eta)\right)=-C_{k}(\theta+\eta) \geq-C_{k}(\theta)-C_{k}(\eta)$, implies that $k$ is a liquidation time, otherwise the right-hand side is an arbitrage. 
An important consequence of the definition of admissibility is that payoffs attainable with zero wealth have nonpositive value under any consistent price system. In frictionless markets, this is the supermartingale property of the gain process under equivalent martingale measures. Liquidation times allow to carry out a similar argument in the present setting.

First, note the intuitively obvious domination property: executing a given strategy at better (bid and ask) prices achieves a better payoff. The discrete time notation streamlines calculations.

Lemma 4.11 Let $(S, \kappa)$ and $\left(S^{\prime}, \kappa^{\prime}\right)$ satisfy Assumption 3.1 and $\kappa-\kappa^{\prime}-\left|S-S^{\prime}\right| \geq 0$. Then, for all simple strategies $\theta$ :

$$
V^{(S, \kappa)}(\theta) \leq V^{\left(S^{\prime}, \kappa^{\prime}\right)}(\theta)-\sum_{n=1}^{\infty}\left(\hat{\kappa}_{n}-\hat{\kappa}_{n}^{\prime}-\left|\hat{S}_{n}-\hat{S}_{n}^{\prime}\right|\right)\left|\hat{\theta}_{n}-\hat{\theta}_{n-1}\right| \leq V^{\left(S^{\prime}, \kappa^{\prime}\right)}(\theta)
$$

Proof Since $V(\theta)=-C(\theta)$, the claim follows from:

$$
\begin{aligned}
\hat{C}_{\infty}^{(S, \kappa)}(\theta)-\hat{C}_{\infty}^{\left(S^{\prime}, \kappa^{\prime}\right)}(\theta) & =\sum_{n=1}^{\infty}\left(\left(\hat{S}_{n}-\hat{S}_{n}^{\prime}\right)\left(\hat{\theta}_{n}-\hat{\theta}_{n-1}\right)+\left(\hat{\kappa}_{n}-\hat{\kappa}_{n}^{\prime}\right)\left|\hat{\theta}_{n}-\hat{\theta}_{n-1}\right|\right) \\
& \geq \sum_{n=1}^{\infty}\left(\hat{\kappa}_{n}-\hat{\kappa}_{n}^{\prime}-\left|\hat{S}_{n}-\hat{S}_{n}^{\prime}\right|\right)\left|\hat{\theta}_{n}-\hat{\theta}_{n-1}\right|
\end{aligned}
$$

Adapting the definitions of Jouini \& Kallal (1995) and Campi \& Schachermayer (2006) to the present setting, consistent price systems take the following form:

Definition 4.12 Let $(S, \kappa)$ satisfy Assumption 3.1. A Strictly Consistent Price System is a pair $(M, Q)$ of a probability $Q$ equivalent to $P$ and a $Q$-local martingale $M$ lying within the bid-ask spread:

$$
\inf _{t \in[0, T]}\left(\kappa_{t}-\left|S_{t}-M_{t}\right|\right)>0 \quad \text { a.s. }
$$

$(M, Q)$ is a Consistent Price System if the inequality is not necessarily strict.

As announced earlier, the basic property of consistent price systems is to assign nonpositive value to simple admissible strategies starting with no initial capital. In the following Proposition, note that $V^{(M, 0)}(\theta)$ represents the frictionless (i.e. with $\kappa=0$ ) portfolio value with price $M$.

Proposition 4.13 Let $(M, Q)$ be a consistent price system. Then $E_{Q}\left[V^{(M, 0)}(\theta)\right] \leq 0$ for all $\theta \in \mathscr{A}^{s}$.

The proof of this property requires a lemma:

Lemma 4.14 Let $\left(M_{t}\right)_{0 \leq t \leq T}$ be a local martingale, $\theta$ a simple strategy and denote by $N_{n}=\sum_{k=1}^{n} \hat{\theta}_{k}\left(\hat{M}_{k}-\hat{M}_{k-1}\right)$. Then $\left(N_{2 n}\right)_{n \geq 0}$ is a $\hat{\mathscr{F}}_{2 n}$-local martingale. 
Proof First, suppose that $M$ is a true martingale, and $\theta$ is bounded. The claim amounts to $E\left[N_{2 n}-N_{2(n-1)} \mid \hat{\mathscr{F}}_{2(n-1)}\right]=0$. Since $\theta_{\sigma_{n}^{-}}=\theta_{\sigma_{n-1}^{+}}$, it follows that:

$$
\begin{aligned}
N_{2 n}-N_{2(n-1)} & =\theta_{\sigma_{n}^{-}}\left(M_{\sigma_{n}^{-}}-M_{\sigma_{n-1}}\right)+\theta_{\sigma_{n}}\left(M_{\sigma_{n}}-M_{\sigma_{n}^{-}}\right) \\
& =\theta_{\sigma_{n-1}^{+}}\left(M_{\sigma_{n}}-M_{\sigma_{n-1}}\right)+\Delta \theta_{\sigma_{n}} \Delta M_{\sigma_{n}}
\end{aligned}
$$

Thus, the first term has the martingale property:

$$
E\left[\theta_{\sigma_{n-1}^{+}}\left(M_{\sigma_{n}}-M_{\sigma_{n-1}}\right) \mid \hat{\mathscr{F}}_{2(n-1)}\right]=\theta_{\sigma_{n-1}^{+}} E\left[M_{\sigma_{n}}-M_{\sigma_{n-1}} \mid \mathscr{F}_{\sigma_{n-1}}\right]=0
$$

Since $\Delta \theta_{\sigma_{n}}$ is $\mathscr{F}_{\sigma_{n}^{-}}$-measurable, the second term satisfies:

$$
E\left[\Delta \theta_{\sigma_{n}} \Delta M_{\sigma_{n}} \mid \mathscr{F}_{\sigma_{n-1}}\right]=E\left[\Delta \theta_{\sigma_{n}} E\left[\Delta M_{\sigma_{n}} \mid \mathscr{F}_{\sigma_{n}^{-}}\right] \mid \mathscr{F}_{\sigma_{n-1}}\right]
$$

To prove that the right-hand side is zero, consider $A_{n} \in \mathscr{F}_{\sigma_{n}}$ which decomposes the stopping time $\sigma_{n}$ into its totally inaccessible part $\sigma_{n A_{n}}$ and accessible part $\sigma_{n \Omega \backslash A_{n}}$. Since $\Delta \theta_{\sigma_{n}}=0$ a.s. on $A_{n}$, it suffices to show that $E\left[\Delta M_{\sigma_{n}} \mid \mathscr{F}_{\sigma_{n}^{-}}\right]=0$ on $\Omega \backslash A_{n}$ to complete the proof for $\theta$ bounded and $M$ a martingale.

To this end, recall that $\mathscr{F}_{\sigma_{n}-}$ is generated by events of the form $A \cap\left\{t<\sigma_{n}\right\}$ for $A \in \mathscr{F}_{t}$, and that the stopping times $\left(\sigma_{n}\right)_{n}$ satisfy $\{\Delta \theta \neq 0\} \subset \cup_{n} \llbracket \sigma_{n} \rrbracket$. Because $\left(\sigma_{n}\right)_{\Omega \backslash A_{n}}$ is accessible (Jacod \& Shiryaev 2003, 1.2.23?), $\left(\sigma_{n}\right)_{\Omega \backslash A_{n}} \subset \cup_{k} \llbracket \tau_{k} \rrbracket$ for a sequence of predictable times $\left(\pi_{k}\right)_{k}$. Thus,

$$
\{\Delta \theta \neq 0\} \cap \llbracket\left(\sigma_{n}\right)_{\Omega \backslash A_{n}} \rrbracket=\sum_{k} \llbracket \tilde{\pi}_{k} \rrbracket
$$

where $\widetilde{\pi}_{k}:=\left(\pi_{k}\right)_{\Delta \theta_{\pi_{k}} \neq 0}$ are still predictable times because $\left\{\Delta \theta_{\pi_{k}} \neq 0\right\} \in \mathscr{F}_{\pi_{k}-}$.

In addition, $\Delta M_{\sigma_{n}} 1_{A \cap\left\{t<\sigma_{n}\right\}} 1_{\Omega \backslash A_{n}}=\Delta M_{\sigma_{n}} 1_{A \cap\left\{t<\sigma_{n}\right\}} 1_{\left\{\sigma_{n}=\left(\sigma_{n}\right)_{\Omega \backslash A_{n}}\right\}}$ because $\Delta M_{\sigma_{n}}=$ 0 on $\left\{\sigma_{n}=\infty\right\}$. In view of (4.22), it follows that:

$$
\begin{aligned}
\Delta \theta_{\sigma_{n}} \Delta M_{\sigma_{n}} 1_{A \cap\left\{t<\sigma_{n}\right\}} 1_{\Omega \backslash A_{n}} & =\sum_{k} \Delta \theta_{\widetilde{\pi}_{k}} \Delta M_{\tilde{\pi}_{k}} 1_{A \cap\left\{t<\tilde{\pi}_{k}\right\}} 1_{\left\{\tilde{\pi}_{k}=\left(\sigma_{n}\right)_{\Omega \backslash A_{n}}\right\}} \\
& =\sum_{k} \Delta \theta_{\widetilde{\pi}_{k}} \Delta M_{\widetilde{\pi}_{k}} 1_{A \cap\left\{t<\tilde{\pi}_{k}\right\}} 1_{\left\{\tilde{\pi}_{k} \leq\left(\sigma_{n}\right)_{\Omega \backslash A_{n}}\right\}} 1_{\left\{\tilde{\pi}_{k} \geq\left(\sigma_{n}\right)_{\Omega \backslash A_{n}}\right\} \cap\left\{\Delta \theta_{\sigma_{n}} \neq 0\right\}}
\end{aligned}
$$

But $\left(A \cap\left\{t<\widetilde{\pi}_{k}\right\}\right) \in \mathscr{F}_{\widetilde{\pi}_{k}-}$ by definition, and $\left\{\widetilde{\pi}_{k} \leq\left(\sigma_{n}\right)_{\Omega \backslash A_{n}}\right\} \in \mathscr{F}_{\widetilde{\pi}_{k}-}$ because $\left\{\left(\sigma_{n}\right)_{\Omega \backslash A_{n}}<\right.$ $\left.\tilde{\pi}_{k}\right\} \in \mathscr{F}_{\widetilde{\pi}_{k}-}$. Moreover, since $\theta_{\widetilde{\pi}_{k}}$ is $\mathscr{F}_{\tilde{\pi}_{k}-}$ measurable, (4.22) implies that:

$$
\left\{\tilde{\pi}_{k} \geq\left(\sigma_{n}\right)_{\Omega \backslash A_{n}}\right\} \cap\left\{\Delta \theta_{\sigma_{n}} \neq 0\right\}=\cup_{j}\left\{\tilde{\pi}_{j} \leq \tilde{\pi}_{k}\right\} \in \mathscr{F}_{\tilde{\pi}_{k}-}
$$

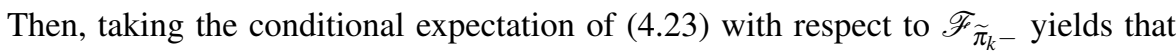
$E\left[\Delta \theta_{\sigma_{n}} \Delta M_{\sigma_{n}} 1_{A \cap\left\{t<\sigma_{n}\right\}} 1_{\Omega \backslash A_{n}}\right]=0$, whence $E\left[\Delta \theta_{\sigma_{n}} \Delta M_{\sigma_{n}} \mid \mathscr{F}_{\sigma_{n}-}\right]=0$.

In general, take a sequence of stopping times $\tau_{j} \uparrow \infty$ localizing $M$, such that $\theta^{\tau_{j}}$ is bounded for each $j$. Since, for a.e. $\omega, \tau_{j}(\omega)<T$ only for finitely many $j$, assume without loss of generality that $\left(\tau_{j}\right)_{j \geq 1} \subset\left(\sigma_{j}\right)_{j \geq 1}$. Then it follows that there are $\hat{\mathscr{F}}$ stopping times $\hat{\tau}_{j}$, taking only even values s.t. $\left(N_{2 n}\right)_{n \geq 0}$ is a $\hat{\mathscr{F}}$-martingale. The claim follows. 
Remark 4.15 Note that $\left(N_{k}\right)_{k \geq 1}$ may not be $\hat{\mathscr{F}}$-martingale, because on the totally inaccessible part of $\sigma_{n}$ the equality $E\left[\Delta M_{\sigma_{n}} \mid \mathscr{F}_{\sigma_{n}^{-}}\right]=0$ may fail.

Proof of Proposition 4.13 Since $\theta$ is simple admissible, by Definition 4.4 it allows a sequence $\left({ }^{k} \theta\right)_{k \geq 1}$ of liquidation strategies satisfying $V^{(S, \kappa)}\left({ }^{k} \theta\right) \geq-x$. Furthermore, by Lemma 4.11:

$$
V^{(M, 0)}\left({ }^{k} \theta\right) \geq V^{(S, \kappa)}\left({ }^{k} \theta\right) \geq-x .
$$

Since $(M, 0)$ is a frictionless, arbitrage-free market for simple strategies, Proposition 4.8 implies admissibility in the frictionless sense:

$$
\sum_{n=1}^{j}{ }^{k} \hat{\theta}_{n-1}\left(\hat{M}_{n}-\hat{M}_{n-1}\right) \geq-x \quad \text { a.s. for all } j, k \geq 1 \text {. }
$$

and as $k \uparrow \infty$ :

$$
N_{2 j}:=\sum_{n=1}^{2 j} \hat{\theta}_{n-1}\left(\hat{M}_{n}-\hat{M}_{n-1}\right) \geq-x \quad \text { a.s. } \quad \text { for all } j \geq 1 .
$$

Take a localizing sequence $\left(\tau_{j}\right)_{j \geq 1}$ for $\left(N_{2 n}\right)_{n \geq 0}$. Lemma 4.14 implies that $E\left[N_{2 j}^{\tau_{j}}\right] \leq 0$ for all $j$, and Fatou's lemma concludes the proof.

The rest of this section shows that both left and right transactions are necessary in Definition 3.3. Two examples make this point, showing that L-simple (simple and left-continuous) admissible strategies cannot approximate R-simple (simple and right-continuous) admissible strategies, and viceversa. Thus, simple strategies must include both types of transactions. Transaction costs are inessential in both examples, hence we set $\kappa=0$.

Example 4.16 Consider the parametric family of densities $f_{\theta}(x)=1_{[-1,1]} \frac{\theta}{e^{\theta}-e^{-\theta}} e^{\theta x}$, which correspond to Esscher transforms of a uniform distribution on $[-1,1]$. All densities in this family are supported by $[-1,1]$, and the expectations span the interval $(-1,1)$ as the parameter $\theta$ varies in $(-\infty, \infty)$.

Now define a sequence of random variables $\left(\varepsilon_{n}\right)_{n \geq 1}$ by setting the density of $\varepsilon_{1}$ equal to $f_{0}$ (i.e. uniformly distributed on $[-1,1]$ ) and the density of $\varepsilon_{n+1}$, conditional on $\mathscr{G}_{n}:=\sigma\left(\left(\varepsilon_{k}\right)_{k \leq n}\right)$, equal to $f_{\theta_{n}}$, where $\theta_{n}\left(\varepsilon_{n}\right)$ is chosen such that $E\left[\varepsilon_{n+1} \mid \mathscr{G}_{n}\right]=\varepsilon_{n}$. Since $\left(\varepsilon_{n}\right)_{n \geq 1}$ is a bounded martingale by construction, it converges a.s. to some random variable $\varepsilon_{\infty}$ whose support contains $\{-1,1\}$ (even conditionally on $\mathscr{G}_{n}$, for each $n \in \mathbb{N}$, a.s.).

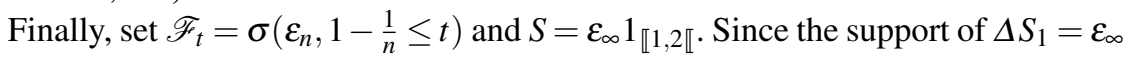
contains $\{-1,1\}$ conditionally on $\mathscr{F}_{t}$ for all $t \in(0,1)$, any L-simple approximation of the R-simple strategy $\theta=\frac{1}{\varepsilon_{\infty}} 1_{\llbracket 1,2 \llbracket}$ can be at best 1 -admissible. But $\theta$ is an arbitrage, hence 0 -admissible. Since the definition of $x$-admissibility in section 4 will require that a general $x$-admissible strategy should be approximated by $x+1 / n$-admissible simple strategies, the present example shows that it is impossible to consider L-simple admissible strategies alone. 


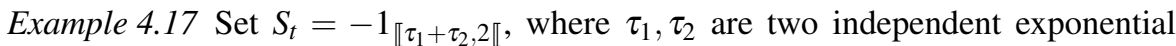
random variables with parameter $\lambda$. The filtration $\mathscr{F}_{t}$ is generated by $\tau_{1}$ and $\tau_{1}+\tau_{2}$ :

$$
\mathscr{F}_{t}:=\sigma\left(\tau_{1} \wedge t,\left(\tau_{1}+\tau_{2}\right) \wedge t\right)
$$

The L-simple strategy $\theta=1_{\rrbracket 1 / 2, \tau_{1} \rrbracket}$ has zero payoff, hence it is 0 -admissible. Consider any R-simple admissible strategy $\eta$, and denote by $\tau_{1}^{\prime}=\inf \left\{t>\tau_{1}: \eta_{t} \neq \eta_{\tau_{1}^{-}}\right\}$. $P\left(\eta_{\tau_{1}^{-}}=\eta_{\tau_{1}}\right)=1$ since $\tau_{1}$ is totally inaccessible, and $P\left(\tau_{1}^{\prime}>\tau_{1}\right)=1$ by rightcontinuity. Observe that $P\left(\tau_{1}+\tau_{2}<\tau_{1}^{\prime}\right)>0$. Indeed, by contradiction suppose that $\tau_{1}^{\prime} \leq \tau_{1}+\tau_{2}$ a.s. Then $\tau_{1}^{\prime}$ would be $\mathscr{F}_{\tau_{1}}$-measurable, i.e. $\tau_{1}^{\prime}=u\left(\tau_{1}\right)$ (cf. Dellacherie $\&$ Meyer (1978, Theorem 105)), and this implies that:

$$
0=P\left(\tau_{1}^{\prime}>\tau_{1}+\tau_{2} \mid \tau_{1}\right)=P\left(\tau_{2}<u\left(\tau_{1}\right)-\tau_{1} \mid \tau_{1}\right)=1-e^{-\lambda\left(u\left(\tau_{1}\right)-\tau_{1}\right)}>0
$$

which is absurd. But $\eta_{\tau_{1}+\tau_{2}}=\eta_{\tau_{1}^{-}}$on the event $\left\{\tau_{1}+\tau_{2}<\tau_{1}^{\prime}\right\}$. Since $\theta_{\tau_{1}^{-}}=1$, it follows that any R-simple approximation is at best 1 -admissible.

\section{5 (RNFLVR) and Finite Variation Strategies}

Let $\mathscr{P}_{V}$ denote the set of predictable finite variation processes. For each $\theta \in \mathscr{P}_{V}$, $\|\theta\|$ denotes the corresponding pathwise total variations (see Appendix A.2 for details). The cost of a predictable finite variation process $\theta$ is defined in terms of the predictable Stieltjes integral constructed in the Appendix:

$$
C(\theta)_{t}=\int_{[0, t]} S d \theta+\int_{[0, t]} \kappa d\|\theta\| \quad \text { a.s., } \quad V(\theta):=-C(\theta) .
$$

A general admissible strategy is now defined as one that is approximated arbitrarily well with simple admissible strategies. The cost of such a strategy is the limit of the cost of the approximations.

Given a predictable process $\theta$ and a random partition $\Sigma$, the $\Sigma$-approximation of $\theta$ is the simple strategy

$$
\theta^{\Sigma}=\sum_{n \geq 1}\left(\theta_{\sigma_{n}} 1_{\llbracket \sigma_{n} \rrbracket}+\theta_{\sigma_{n}^{+}} 1_{\rrbracket \sigma_{n}, \sigma_{n+1} \llbracket}\right)
$$

Definition 5.1 A predictable process $\theta$ of finite variation is an admissible strategy if, for any $\varepsilon>0$ there exists a simple strategy $\theta^{\varepsilon} \in \mathscr{A}^{s}$ such that $\left|\theta^{\varepsilon}-\theta\right| \leq \varepsilon$ and $\left|V\left(\theta^{\varepsilon}\right)-V(\theta)\right| \leq \varepsilon$. Denote the space of admissible strategies by $\mathscr{A}$.

\section{Definition 5.2}

i) $(S, \kappa)$ satisfies (NFLVR) if, for any sequence $\left(\theta^{n}\right)_{n \geq 1}$ such that $\theta^{n} \in \mathscr{A}_{1 / n}^{s}$ and $V\left(\theta^{n}\right)$ converges a.s. to some limit $V \in[0, \infty]$ a.s., then $V=0$ a.s.

ii) $(S, \kappa)$ satisfies (RNFLVR) if there exists a pair $\left(S^{\prime}, \kappa^{\prime}\right)$ satisfying (NFLVR) and such that the bid-ask spread of $\left(S^{\prime}, \kappa^{\prime}\right)$ is a.s. strictly contained within that of $(S, \kappa)$, pathwise uniformly:

$$
\inf _{t \in[0, T]}\left(\kappa_{t}-\kappa_{t}^{\prime}-\left|S_{t}-S_{t}^{\prime}\right|\right)>0 \quad \text { a.s. }
$$


Remark 5.3 Equation (5.3) is equivalent to:

$$
\inf _{t \in[0, T]}\left((S+\kappa)_{t}-\left(S^{\prime}+\kappa^{\prime}\right)_{t}\right)>0 \quad \text { and } \quad \inf _{t \in[0, T]}\left(\left(S^{\prime}-\kappa^{\prime}\right)_{t}-(S-\kappa)_{t}\right)>0 \quad \text { a.s. }
$$

which means that the inner bid and ask prices never touch their outer counterparts. Observe also that (RNFLVR) implies the efficient friction condition:

$$
\inf _{t \in[0, T]} \kappa_{t}>0 \quad \text { a.s. }
$$

so the bid-ask spread is always strictly positive, in pathwise uniform sense.

The next Lemma follows from Definition 5.2 with essentially the same argument as Proposition 3.1 in Delbaen \& Schachermayer (1994):

Lemma 5.4 If $(S, \kappa)$ satisfies (NFLVR), then $\mathscr{C}_{x}^{s}:=\left\{V(\theta): \theta \in \mathscr{A}_{x}^{s}\right\}-L_{+}^{0}$ is bounded from above in $L^{0}$ for all $x>0$, i.e. $\lim _{n \rightarrow \infty} \sup _{F \in \mathscr{C}_{x}^{s}} P(F>n)=0$.

Proof It suffices to prove the claim for $x=1$. By contradiction, suppose there exists $\alpha>0$ and a sequence $\left(\theta^{n}\right)_{n \geq 1} \subset \mathscr{A}_{1}^{s}$ such that $P\left(V\left(\theta^{n}\right)>n\right)>\alpha$ for all $n$. Then the sequence $\left(\eta^{n}\right)_{n \geq 1}$ defined by $\eta_{t}^{n}=\theta_{t}^{n} / n$ satisfies $\eta^{n} \in \mathscr{A}_{1 / n}^{s}$ for all $n \geq 1$, and $P\left(V\left(\eta^{n}\right)>1\right)>\alpha$. Up to a sequence of convex combinations (Lemma B.1), $V\left(\eta^{n}\right)$ converges to $V_{\infty} \in[0, \infty]$ a.s. The (NFLVR) condition implies that $V_{\infty}=0$. It follows that $n^{-1}+\min \left(V\left(\eta^{n}\right), 1\right)$ converges a.s. to $f=0$. On the other hand, the Lebesgue theorem yields $E f=\lim _{n} E \min \left(V\left(\eta^{n}\right), 1\right)$ where

$$
E\left[n^{-1}+\min \left(V\left(\eta^{n}\right), 1\right)\right] \geq E\left[n^{-1}+\min \left(V\left(\eta^{n}\right), 1\right)\right] 1_{V\left(\eta^{n}\right)>1} \geq \alpha
$$

hence a contradiction.

The previous lemma, combined with the domination property (Lemma 4.11), implies that the total variations of $x$-admissible strategies are bounded in probability. Henceforth, $\|\theta\|_{T}$ denotes the pathwise total variation of the process $\theta$ on $[0, T]$.

Lemma 5.5 Let $(S, \kappa)$ satisfy (RNFLVR). Then $\left\{\|\theta\|_{T}: \theta \in \mathscr{A}_{x}^{s}\right\}$ is bounded in $L^{0}$ for all $x>0$.

Proof Rearranging (4.17), for any $\theta \in \mathscr{A}_{x}^{s}$ the following holds:

$\|\theta\|_{T} \inf _{n \geq 1}\left(\hat{\kappa}_{n}-\hat{\kappa}_{n}^{\prime}-\left|\hat{S}_{n}-\hat{S}_{n}^{\prime}\right|\right) \leq \sum_{n=1}^{\infty}\left(\hat{\kappa}_{n}-\hat{\kappa}_{n}^{\prime}-\left|\hat{S}_{n}-\hat{S}_{n}^{\prime}\right|\right)\left|\hat{\theta}_{n}-\hat{\theta}_{n-1}\right| \leq x+V^{\left(S^{\prime}, \kappa^{\prime}\right)}(\theta)$

Note that $\inf _{n \geq 1}\left(\hat{\kappa}_{n}-\hat{\kappa}_{n}^{\prime}-\left|\hat{S}_{n}-\hat{S}_{n}^{\prime}\right|\right) \geq Y:=\inf _{t \in[0, T]}\left(\kappa_{t}-\kappa_{t}^{\prime}-\left|S_{t}-S_{t}^{\prime}\right|\right)>0$. Lemma 5.4 implies that the set $\left\{\|\theta\|_{T} Y: \theta \in \mathscr{A}_{x}^{s}\right\}$ and hence also $\left\{\|\theta\|_{T}: \theta \in \mathscr{A}_{x}^{s}\right\}$ are bounded in $L^{0}$.

Denote by $\mathscr{C}_{x}=\left\{V(\theta)_{T}: \theta \in \mathscr{A}\right\}-L_{+}^{0}$ the set of claims dominated by outcomes of admissible portfolios. Recall that $\left(X_{n}\right)_{n \geq 1} \subset L^{0}$ Fatou converges to $X$ if $X_{n}$ converges to $X$ a.s., and $X_{n} \geq-c$ a.s. for all $n$ and some $c>0$. 
Proposition 5.6 Under (RNFLVR), $\mathscr{C} \cap\left\{Z:\|Z\|_{\infty} \leq x\right\}$ is Fatou closed for each $x>$ 0 .

Proof Let $\left(X_{n}\right)_{n \geq 1} \subset \mathscr{C}$ Fatou converge to $X$. It suffices to find some $\theta \in \mathscr{A}$ such that $V(\theta) \geq X$ a.s. By assumption, $X_{n}=V\left(\theta^{n}\right)-f_{n} \geq-x$ for some $\theta^{n} \in \mathscr{A}$ and $f_{n} \geq 0$ a.s. In particular, $V\left(\theta^{n}\right) \geq-x$. By assumption, there exists $\tilde{\theta}^{n} \in \mathscr{A}^{s}$ such that $\left|\tilde{\theta}^{n}-\theta^{n}\right| \leq n^{-1}$ and $\left|V\left(\tilde{\theta}^{n}\right)-V\left(\theta^{n}\right)\right| \leq n^{-1}$, and therefore $\tilde{\theta}^{n} \in \mathscr{A}_{x+\frac{1}{n}}^{s}$ by Proposition 4.9. Since $\left(\tilde{\theta}^{n}\right)_{n \geq 1} \subset \mathscr{A}_{x+\frac{1}{n}}^{s},\left(\left\|\tilde{\theta}^{n}\right\|_{T}\right)_{n \geq 1}$ is bounded in $L^{0}$ by Lemma 5.5. Thus, by Lemma B.4, up to a sequence of convex combinations $\tilde{\theta}^{n}$ converge a.s. to some $\theta \in$ $\mathscr{P}_{V}, \tilde{\theta}_{+}^{n} \rightarrow \theta_{+}$and $\left\|\tilde{\theta}^{n}\right\|$ converges pointwise to $\|\theta\|$. To show that $\theta \in \mathscr{A}_{x}$, consider $\varepsilon>0$, and the strategy $\theta^{\varepsilon}$ given by Corollary A.13, which satisfies $\left|\theta^{\varepsilon}-\theta\right| \leq \varepsilon$ and $\left|V\left(\theta^{\varepsilon}\right)-V(\theta)\right| \leq \varepsilon$. Consider a given partition $\Sigma$ defining $\theta^{\varepsilon}$. By assumption (cf. remark 4.5), for any stopping time $\sigma \in \Sigma$, there exists liquidation times $\sigma^{n *} \geq \sigma$ and $\sigma_{+}^{n *} \geq \sigma$ verifying

$$
\begin{aligned}
& V_{\sigma}\left(\tilde{\theta}^{n}\right)+\tilde{\theta}_{\sigma}^{n}(S \pm \kappa) \sigma^{n *}+\left(x+\frac{1}{n}\right) \geq 0 \\
& V_{\sigma+}\left(\tilde{\theta}^{n}\right)+\tilde{\theta}_{\sigma+}^{n}(S \pm \kappa)_{\sigma_{+}^{n *}}+\left(x+\frac{1}{n}\right) \geq 0 .
\end{aligned}
$$

Now, define the stopping times $\sigma^{*}:=\inf _{n} \sigma^{n *}$ and $\sigma_{+}^{*}:=\inf _{n} \sigma_{+}^{n *}$. Notice that a.s. there exists a decreasing subsequence such that $\sigma^{n *} \rightarrow \sigma^{*}$ and $\sigma_{+}^{n *} \rightarrow \sigma_{+}^{*}$. Also, since $S$ and $\kappa$ are càdlàg, Theorem A.9 iii) implies that:

$$
\begin{aligned}
& V(\theta)_{\sigma}+\theta_{\sigma}(S \pm \kappa)_{\sigma^{*}}+x \geq 0 \\
& V(\theta)_{\sigma+}+\theta_{\sigma+}(S \pm \kappa) \sigma_{+}^{*}+x \geq 0 .
\end{aligned}
$$

Further, define the finer extended partition $\Sigma^{*}:=\Sigma \cup\left\{\sigma^{*}, \sigma_{+}^{*}: \sigma \in \Sigma\right\}$. By definition, $\theta_{\sigma}=\theta_{\sigma}^{\varepsilon}$ and $\theta_{\sigma_{+}}=\theta_{\sigma_{+}}^{\varepsilon}$ if $\sigma \in \Sigma$. Thus, from (5.4) it follows that:

$$
\begin{aligned}
& V\left(\theta^{\varepsilon}\right)_{\sigma}+\theta_{\sigma}^{\varepsilon}(S \pm \kappa)_{\sigma^{*}}+x+\varepsilon \geq 0 \\
& V\left(\theta^{\varepsilon}\right)_{\sigma+}+\theta_{\sigma+}^{\varepsilon}(S \pm \kappa)_{\sigma_{+}^{*}}+x+\varepsilon \geq 0 .
\end{aligned}
$$

These inequalities shows that $\theta^{\varepsilon} \in \mathscr{A}_{x+\varepsilon}^{s}$. Indeed, the statements of Definition 4.4 hold respectively with $\widehat{\Sigma}$, the partition defining the strategy $\widehat{\theta^{\varepsilon}}$, and the finer extended partition $\widehat{\Sigma^{*}}$. Now, $\theta^{\varepsilon} \in \mathscr{A}^{s}$ yields that $\theta \in \mathscr{A}$, and $V\left(\tilde{\theta}^{n}\right)+n^{-1} \geq X_{n}$ implies that $V(\theta) \geq X$.

The following Proposition is needed for the implication $(S C P S) \Rightarrow(R N F L V R)$.

\section{Proof of Main Result}

This section proves Theorem 1.1. The proof employs the usual separation method, combined with the following argument, in the spirit of Jouini \& Kallal (1995), which is proved after the main theorem. 
Let $(S, \kappa)$ satisfy (RNFLVR), and let $\left(S^{\prime}, \kappa^{\prime}\right)$ be its corresponding bid-ask pair. Then, the auxiliary pair $(\hat{S}, \hat{\kappa}):=([S+\tilde{S}] / 2,[\kappa+\tilde{\kappa}] / 2)$ satisfies (RNFLVR). Indeed:

$\inf _{t \in[0, T]}\left(\tilde{\kappa}_{t}-\hat{\kappa}_{t}-\left|\tilde{S}_{t}-\hat{S}_{t}\right|\right)=\inf _{t \in[0, T]}\left(\kappa_{t}-\hat{\kappa}_{t}-\left|S_{t}-\hat{S}_{t}\right|\right)=\inf _{t \in[0, T]} \frac{\left(\kappa_{t}-\tilde{\kappa}_{t}-\left|S_{t}-\tilde{S}_{t}\right|\right)}{2}>0$

Lemma 6.1 If $Q$ is equivalent to $P$ and $E_{Q}\left[V^{(\hat{S}, \hat{\kappa})}(\theta)\right] \leq 0$ for all $\theta \in \mathscr{A}^{(\hat{S}, \hat{\kappa})}$ with $V^{(\hat{S}, \hat{\kappa})}(\theta) \in L^{\infty}$, then there exists a $Q$-local martingale $M$ such that $(M, Q)$ is a strictly consistent price system for $(S, \kappa)$.

Proof of Theorem 1.1 (SCPS) $\Rightarrow$ (RNFLVR): It suffices to check that $\tilde{S}=M$ and $\tilde{\kappa}=0$ satisfies (NFLVR). First, note that (5.3) follows immediately:

$$
\inf _{t \in[0, T]}\left(\kappa_{t}-\tilde{\kappa}_{t}-\left|S_{t}-\tilde{S}_{t}\right|\right)=\inf _{t \in[0, T]}\left(\kappa_{t}-\left|S_{t}-M_{t}\right|\right)>0 \quad \text { a.s. }
$$

To check that (NFLVR) holds, let $\left(\theta^{n}\right)_{n \geq 1}$ be such that $\theta^{n} \in \mathscr{A}_{1 / n}^{s}$ and $V^{(M, 0)}\left(\theta^{n}\right)$ converge a.s. to some $V$. Proposition 4.13 implies that $E_{Q}\left[V^{(M, 0)}\left(\theta^{n}\right)\right] \leq 0$. Thus, by Fatou's lemma $E_{Q}[V] \leq 0$ and $V=0$ a.s.

$(\mathrm{RNFLVR}) \Rightarrow$ (SCPS): Consider the convex set $\mathscr{C}$ (with respect to $(\hat{S}, \hat{\kappa}))$. Then the convex $C_{x}:=\mathscr{C} \cap\left\{Z:\|Z\|_{\infty} \leq x\right\}$ is Fatou closed for $x>0$ by Proposition 5.6 and Lemma B.2 implies that $C:=\mathscr{C} \cap L^{\infty}$ is $\sigma\left(L^{\infty}, L^{1}\right)$-closed. By Theorem B.3, there exists a probability $Q$, equivalent to $P$ such that $E_{Q}[C] \leq 0$, and Lemma 6.1 concludes the proof.

The rest of this section proves Lemma 6.1. The arguments are standard, and included only for completeness. The next lemma is due to Jouini \& Kallal (1995) (cf. also Choulli \& Stricker (1998) and Cherny (2007)).

Lemma 6.2 Let $\left(X_{t}\right)_{t \in[0, T]}$ be a supermartingale and $\left(Y_{t}\right)_{t \in[0, T]}$ a submartingale, such that $X \leq Y$ a.s. Then there exists a martingale $\left(M_{t}\right)_{t \in[0, T]}$ such that $X \leq M \leq Y$ a.s.

Proof For finitely many time instants $0 \leq t_{0}<\cdots<t_{n} \leq T$, set $M_{t_{0}}=Y_{t_{0}}$ and recursively define $M_{t_{n+1}}=\alpha_{n} X_{t_{n+1}}+\left(1-\alpha_{n}\right) Y_{t_{n+1}}$, where the $\mathscr{F}_{t_{n}}$-measurable $\alpha_{n}$ satisfies:

$$
M_{t_{n}}=\alpha_{n} E\left[X_{t_{n+1}} \mid \mathscr{F}_{t_{n}}\right]+\left(1-\alpha_{n}\right) E\left[Y_{t_{n+1}} \mid \mathscr{F}_{t_{n}}\right]
$$

The proof of the induction only requires that $M_{t_{n}} \in\left[E\left(X_{t_{n+1}} \mid \mathscr{F}_{t_{n}}\right) ; E\left(Y_{t_{n+1}} \mid \mathscr{F}_{t_{n}}\right)\right]$ but this is important to get the existence of the coefficients. Then $M$ is a martingale for the filtration $\left(\mathscr{F}_{t_{i}}\right)_{0 \leq i \leq n}$ and $X_{t_{i}} \leq M_{t_{i}} \leq Y_{t_{i}}$ for $i=0, \ldots, n$. From the discrete case just considered, each dyadic partition $D_{n}=\left\{k T / 2^{n}: 0 \leq k \leq 2^{n}\right\}$ yields a martingale $M^{n}$ with respect to the discrete filtration $\left(\mathscr{F}_{t}\right)_{t \in D_{n}}$, such that:

$$
X_{t} \leq M_{t}^{n} \leq Y_{t} \quad \text { for all } t \in D_{n}
$$

In particular, $X_{T} \leq M_{T}^{n} \leq Y_{T}$ for all $n \geq 1$, therefore $\left(M_{T}^{n}\right)_{n \geq 1}$ is bounded in $L^{1}$, and by Komlós Theorem it converges up to a sequence of convex combinations to some random variable $M$ almost surely and in $L^{1}$ (due to the integrability of $Y_{T}$ ). Then define the martingale $M_{t}=E\left[M_{T} \mid \mathscr{F}_{t}\right]$, and passing to the limit in (6.1) as $n \rightarrow \infty$ yields $X_{t} \leq M_{t} \leq Y_{t}$ a.s. for all $t \in[0, T]$. 
The next Lemma extends Theorem 4.5 in Cherny (2007) to the present setting:

Lemma 6.3 Let $\left(X_{t}\right)_{t \in[0, T]}$ and $\left(Y_{t}\right)_{t \in[0, T]}$ be two càdlàg bounded processes. The following conditions are equivalent:

i) There exists a càdlàg martingale $\left(M_{t}\right)_{t \in[0, T]}$ such that:

$$
X \leq M \leq Y \quad \text { a.s. }
$$

ii) For all stopping times $\sigma, \tau$ such that $0 \leq \sigma \leq \tau \leq T$ a.s., the following hold:

$$
E\left[X_{\tau} \mid \mathscr{F}_{\sigma}\right] \leq Y_{\sigma} \quad \text { and } \quad E\left[Y_{\tau} \mid \mathscr{F}_{\sigma}\right] \geq X_{\sigma} \quad \text { a.s. }
$$

Proof

i) $\Rightarrow i i):(6.2)$ and the optional sampling theorem imply that:

$$
E\left[X_{\tau} \mid \mathscr{F}_{\sigma}\right] \leq E\left[M_{\tau} \mid \mathscr{F}_{\sigma}\right]=M_{\sigma} \leq Y_{\sigma}
$$

and the second equation in (6.3) follows similarly.

$i i) \Rightarrow i$ ): Denoting by $\mathscr{O}_{t}$ the set of stopping times with values in the interval $[t, T]$, define the auxiliary processes:

$$
X_{t}^{\prime}=\operatorname{ess}_{\sup } \operatorname{se}_{\tau} E\left[X_{\tau} \mid \mathscr{F}_{t}\right] \quad \text { and } \quad Y_{t}^{\prime}=\operatorname{ess} \inf _{\tau \in \mathscr{O}_{t}} E\left[Y_{\tau} \mid \mathscr{F}_{t}\right]
$$

Note that $\sigma 1_{A}+\tau 1_{\Omega \backslash A} \in \mathscr{O}_{t}$ if $\sigma, \tau \in \mathscr{O}_{t}$ and $A \in \mathscr{F}_{t}$. Now, observe that the family $E\left[X_{\tau} \mid \mathscr{F}_{t}\right], \tau \in \mathscr{O}_{t}$ is directed upwards, hence there is a sequence $\tau_{n} \in \mathscr{O}_{t}$ attaining the essential supremum, i.e. $X_{t}^{\prime}=\lim \uparrow_{n} E\left(X_{\tau_{n}} \mid \mathscr{F}_{t}\right)$ for $u<t$. Thus, $X^{\prime}$ is a supermartingale:

$$
E\left(X_{t}^{\prime} \mid \mathscr{F}_{u}\right)=\lim \uparrow_{n} E\left(X_{\tau_{n}} \mid \mathscr{F}_{u}\right) \leq X_{u}^{\prime} .
$$

Likewise, $Y^{\prime}$ is a submartingale, and they both admit càdlàg versions (this follows as in Proposition 4.3 in Kramkov (1996)). ii) implies that, for all $\sigma, \tau \in \mathscr{O}_{t}$ :

$$
\begin{aligned}
& E\left[X_{\tau} \mid \mathscr{F}_{t}\right]-E\left[Y_{\sigma} \mid \mathscr{F}_{t}\right]=E\left[E\left[X_{\tau}-Y_{\sigma} \mid \mathscr{F}_{\tau \wedge \sigma}\right] \mid \mathscr{F}_{t}\right]= \\
& \quad=E\left[\left(X_{\tau}-E\left[Y_{\sigma} \mid \mathscr{F}_{\tau}\right]\right) 1_{\{\tau \leq \sigma\}}+\left(E\left[X_{\tau} \mid \mathscr{F}_{\sigma}\right]-Y_{\sigma}\right) 1_{\{\sigma<\tau\}} \mid \mathscr{F}_{t}\right] \leq 0 \quad \text { a.s. }
\end{aligned}
$$

and hence $X_{t}^{\prime} \leq Y_{t}^{\prime}$ a.s. for all $t \in[0, T]$. Lemma 6.2 concludes the proof.

Proof of Lemma 6.1 Take $\tau_{n}$ a sequence of stopping times with $\tau_{n} \uparrow \infty$ s.t. $\hat{S}^{\tau_{n}}, \hat{\kappa}^{\tau_{n}}$ are bounded processes. Then, for any stopping times $\sigma<\tau$ and $A \in \mathscr{F}_{\sigma}$ the strategy $\theta= \pm 1_{A \cap \rrbracket \sigma, \tau \rrbracket}$ is admissible for $\left(\hat{S}^{\tau_{n}}, \hat{\kappa}^{\tau_{n}}\right)$. For brevity, write $\check{S}:=\hat{S}^{\tau_{n}}$ and $\breve{\kappa}:=\hat{\kappa}^{\tau_{n}}$ in the next few lines. For each $n$, (which is implicit in the notation):

$$
E_{Q}\left[\left((\check{S}-\check{\kappa})_{\tau}-(\check{S}+\check{\kappa})_{\sigma}\right) 1_{A}\right] \leq 0, \quad E_{Q}\left[\left((\check{S}+\check{\kappa})_{\tau}-(\check{S}-\check{\kappa})_{\sigma}\right) 1_{A}\right] \leq 0
$$

Since these equations hold for any $A \in \mathscr{F}_{\sigma}$, it follows that:

$$
E_{Q}\left[(\check{S}+\check{\kappa})_{\tau} \mid \mathscr{F}_{\sigma}\right] \leq(\check{S}-\check{\kappa})_{\sigma}, \quad E_{Q}\left[(\check{S}-\check{\kappa})_{\tau} \mid \mathscr{F}_{\sigma}\right] \leq(\check{S}+\check{\kappa})_{\sigma}
$$

and Lemma 6.3 implies the existence of a $Q$-martingale $M$ such that:

$$
\left|\hat{S}_{t}^{\tau_{n}}-M_{t}^{n}\right| \leq \hat{\kappa}_{t}^{\tau_{n}} \quad \text { a.s. for all } t \in[0, T]
$$


By the construction in Lemma $6.2, M_{t}^{n}$ and $M_{t}^{n+1}$ coincide on $\left[0, \tau_{n}\right]$. Thus, the local martingale $M_{t}$ on $[0, T]$ resulting from this construction satisfies:

$$
\left|\hat{S}_{t}-M_{t}\right| \leq \hat{\kappa}_{t}, \quad t \in[0, T]
$$

Finally, (6) implies the required property:

$$
\inf _{t \in[0, T]}\left(\kappa_{t}-\left|S_{t}-M_{t}\right|\right) \geq \inf _{t \in[0, T]}\left(\kappa_{t}-\hat{\kappa}_{t}-\left|\hat{S}_{t}-S_{t}\right|\right)>0
$$

\section{Conclusion}

This paper proves the Fundamental Theorem of Asset Pricing under transaction costs in continuous time for potentially discontinuous - but locally bounded - prices.

The treatment focuses on a single risky asset mainly to ease notation. With the exception of Proposition 4.9, these results hold virtually unchanged for models with several assets trading against a common numéraire. The extension of Proposition 4.9 to several risky assets becomes exceedingly complicated, as each risky asset may require a different liquidation time. Such an extension is not treated here.

The main conceptual difference between this model (which follows Jouini \& Kallal (1995)) and the pure exchange models initiated by Kabanov (1999) is in the role of the numéraire. Exchange models are by construction numéraire free and unlike frictionless models - define admissibility symmetrically with respect to all assets. This symmetry is possible because in such models relative prices are always positive.

In the bid-ask model considered here, the numéraire is the only measure of value, since prices can be positive as well as negative. A trading strategy is admissible when its eventual liquidation leads to a bounded loss. Boundedness crucially depends on the choice of numéraire, as in the frictionless theory.

\section{A Predictable Stieltjes integral}

The usual Stieltjes integral $\int S d \theta$ is well-defined for continuous integrands $S$ and integrators $\theta$ of finite variation. This subsection defines an extension of the Stieltjes integral to càdlàg integrands, which makes the integral operator continuous with respect to the pointwise convergence of integrators.

The discussion is divided into three parts: the first subsection recalls some properties of predictable finite variation processes, available in most textbooks under the extra assumption of càdlàg processes, relaxed here to làdlàg. The second subsection defines the integral, and establishes its Lebesgue- and Fatou-type properties. Since the integral is defined pathwise, the first two subsections are probability-free, requiring only a filtered measurable space with a right-continuous filtration. Thus, the special case of deterministic integrand and integrator is included in this setting. The third subsection establishes an approximation result for the predictable integral, which requires an underlying probability measure, since it relies on the decomposition of a 
stopping time into its accessible and totally inaccessible parts. This part requires a filtered probability space satisfying the usual conditions.

\section{A.1 Predictable finite variation processes}

This subsection recalls some properties of finite variation processes, dropping the extra assumption of right-continuity, which appears in most textbooks.

Consider a measurable space $(\Omega, \mathscr{F})$, endowed with a right-continuous filtration $\left(\mathscr{F}_{t}\right)_{t \geq 0}$ such that $\mathscr{F}_{\infty}=\mathscr{F}$. On the set $\Omega \times \mathbb{R}_{+}$, the predictable $\sigma$-algebra is generated by the class of adapted and left-continuous processes. Denote by $\mathscr{P}_{V}$ the class of predictable processes with pathwise finite variation; by $\mathscr{D}$ the class of adapted càdlàg processes and by $\mathscr{L}$ the class of adapted làglàd (right-limited and left-limited) processes. Since a function of finite variation has right and left limits at all points, it follows that $\mathscr{P}_{V} \subset \mathscr{L}$. A sequence of processes $X^{n}$ converges pointwise to a process $X$ if $X^{n}(\omega, t) \rightarrow X(\omega, t)$ for all $(\omega, t) \in \Omega \times \mathbb{R}_{+}$.

Proposition A.1 Let $\theta \in \mathscr{P}_{V}$ and let $\|\theta\|$ denote its pathwise total variation. Then:

i) $\|\theta\| \in \mathscr{P}_{V}$;

ii) if $\left(\theta^{n}\right)_{n \geq 1} \subset \mathscr{P}_{V}$ converge pointwise to $\theta$, then:

$$
\|\theta\| \leq \liminf _{n \rightarrow \infty}\left\|\theta^{n}\right\| \text { pointwise }
$$

The proof requires a representation of the set of jumps of predictable finite variation processes. The finite-variation property avoids the use of section theorems. Recall that a random set $A \subset \Omega \times[0, \infty)$ is thin if $A \subset \cup_{n>1} \llbracket \tau_{n} \rrbracket$ for a sequence of stopping times $\left(\tau_{n}\right)_{n \geq 1}$.

Lemma A.2 Let $X \in \mathscr{P}_{V}$. Then the sets $\left\{X \neq X_{-}\right\}$and $\left\{X \neq X_{+}\right\}$are thin, and $\left\{X \neq X_{-}\right\}$is predictable.

Proof For $n, p \geq 0$, define the stopping times $S_{n, p}$ by induction as $S_{n, 0}=0$ and:

$$
S_{n, p+1}= \begin{cases}\inf \left\{t>S_{n, p}:\left|X_{t}-X_{S_{n, p}^{+}}\right|>2^{-n}\right\} & \text { for even } p \\ \inf \left\{t \geq S_{n, p}:\left|X_{t}-X_{S_{n, p}}\right|>2^{-n}\right\} & \text { for odd } p\end{cases}
$$

By definition, $S_{n, p}<S_{n, p+2}$. Define also the random sets:

$$
A_{n, p}= \begin{cases}\left\{X_{S_{n, p}} \neq X_{S_{n, p}^{-}}, S_{n, p}<\infty\right\} & \text { for even } p \\ \left\{X_{S_{n, p}^{+}} \neq X_{S_{n, p}}, S_{n, p}<\infty\right\} & \text { for odd } p\end{cases}
$$

and observe that $A_{n, p} \in \mathscr{F}_{S_{n, p}}$. This is obvious for $p$ even, while for $p$ odd the rightcontinuity of the filtration implies that $X_{S_{n, p}^{+}}$is $\mathscr{F}_{S_{n, p}}$-measurable, whence the claim. Then, set $T_{n, p}=S_{n, p} 1_{A_{n, p}}+\infty 1_{\Omega \backslash A_{n, p}}$, which is a stopping time since $A_{n, p} \in \mathscr{F}_{S_{n, p}}$. As $X$ has finite variation, $\lim _{p \rightarrow \infty} S_{n, p}=\infty$ and therefore:

$$
\left.\left\{X \neq X_{-}\right\}=\cup_{n, p \text { even }}\left[T_{n, p}\right]\right] \quad \text { and } \quad\left\{X_{+} \neq X\right\}=\cup_{n, p \text { odd }}\left[\left[T_{n, p}\right]\right],
$$

so the sets $\left\{X \neq X_{+}\right\}$and $\left\{X_{-} \neq X\right\}$ are thin. Also, $X_{-}$is left-continuous, therefore the set $\left\{X \neq X_{-}\right\}$is predictable. 
Proof of Proposition A.1. The proof of $i$ ) is similar to the càdlàg case (Delbaen \& Schachermayer 2006, Theorem 12.2.1). By Lemma A.2, there is an exhausting sequence of stopping times $\left(\sigma_{k}\right)_{k \geq 1}$ for the set of jumps of $\theta$. For a fixed $n \geq 1$, denote by $\Pi_{n}=\left(\tau_{k}\right)_{0 \leq k \leq N_{n}}$ the finite ordered sequence of stopping times obtained from the set $\left(k 2^{-n}\right)_{0 \leq k \leq 2^{n} T} \cup\left(\sigma_{k}\right)_{k \leq n}$, and define the processes:

$$
V_{t}\left(\theta, \Pi_{n}\right)=\sum_{k=1}^{N_{n}}\left|\theta_{\tau_{k} \wedge t}-\theta_{\tau_{k-1} \wedge t}\right|
$$

$V\left(\theta, \Pi_{n}\right)$ is predictable because $\theta$ is, and since $V\left(\theta, \Pi_{n}\right)$ converges to $\|\theta\|$ pointwise, also $\|\theta\|$ is predictable and $i$ ) follows.

For $i i)$, denote by $\Pi=\left(\tau_{k}\right)_{0 \leq k \leq n}$ a finite ordered sequence of stopping times, and define $V(\theta, \Pi)$ as in (A.1). The pointwise convergence of $\left(\theta^{n}\right)_{n \geq 1}$ to $\theta$ implies that:

$$
\lim _{n \rightarrow \infty} V_{t}\left(\theta^{n}, \Pi\right)=V_{t}(\theta, \Pi) \text { for all } t, \Pi
$$

By $i$ ) it follows that $\|\theta\|_{t}=\sup _{\Pi} V_{t}(\theta, \Pi)$, and therefore:

$$
\liminf _{n \rightarrow \infty}\left\|\theta^{n}\right\|_{t}=\liminf _{n \rightarrow \infty} \sup _{\Pi} V_{t}\left(\theta^{n}, \Pi\right) \geq \sup _{\Pi} \lim _{n \rightarrow \infty} V_{t}\left(\theta^{n}, \Pi\right)=\sup _{\Pi} V_{t}(\theta, \Pi)=\|\theta\|_{t}
$$

which proves $i i)$.

\section{A.2 The Predictable Stieltjes Integral}

This section defines the integral $\int_{0}^{t} S d \theta$ for each $\theta \in \mathscr{P}_{V}$ and $S \in \mathscr{D}$. Without additional assumptions on $\theta$ and $S$, such an integral may not exist in the standard Stieltjes sense. A simple example demonstrates the problem:

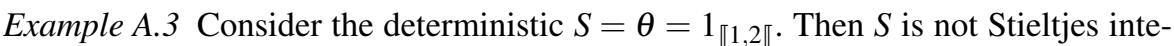
grable with respect to $\theta$, because there are arbitrarily fine subdivisions such that the corresponding Riemann sums are either 0 or 1 . Indeed, some Riemann sums include the jump $\Delta S_{1} \Delta \theta_{1}$, while others do not.

However, $\int S d \theta$ is a Stieltjes integral if $\theta$ is left-continuous:

Lemma A.4 Let $S$ be a càdlàg function and $\theta$ a function of finite variation. Then $\int_{[0, T]} S d \theta_{-}$exists as a Stieltjes integral.

The proof requires a simple Lemma.

Lemma A.5 Let $S$ be a càdlàg (deterministic) function such that $\left|\Delta S_{t}\right| \leq s$ for all $t$. Then for all $\eta>s$ there exists $\delta>0$ such that $\left|S_{u}-S_{t}\right| \leq \eta$ for all $u, t$ such that $|t-u| \leq \delta$.

Proof By the càdlàg assumption, for all $t \in[0, T]$ there exists some $\delta_{t}>0$ such that $\left|S_{u}-S_{t}\right|<(\eta-s) / 2$ for $u \in\left[t, t+\delta_{t}\right)$ and $\left|S_{u}-S_{t^{-}}\right|<(\eta-s) / 2$ for $u \in\left(t-\delta_{t}, t\right)$. Therefore $\left|S_{u}-S_{t}\right|<\eta$ for all $u \in U_{t}=\left(t-\delta_{t}, t+\delta_{t}\right)$. By compactness, the open covering of $\left(U_{t}\right)_{t \in[0, T]}$ admits a finite subcovering $\left(U_{t_{i}}\right)_{i=1}^{n}$, and the thesis follows with $\delta=\min _{1 \leq i \leq n} \delta_{t_{i}}$. 
Proof of Lemma A.4. Recall two basic facts (cf. Wheeden \& Zygmund (1977)). First, the Stieltjes integral $\int f d g$ exists if and only if for all $\varepsilon>0$ there exists a $\delta>0$ such that for any pair of partitions $\Gamma, \Gamma^{\prime}$ with mesh smaller than $\delta$, the corresponding Riemann sums differ by less than $\varepsilon$. Second, if two functions $f$ and $g$ have a finite number of jump discontinuities, then the integrals $\int f d g$ and $\int g d f$ are defined if and only if at each (common) discontinuity point one function is right-continuous and the other is left-continuous.

For a given $\varepsilon>0$, set $J_{t}^{\varepsilon}=\sum_{s \leq t} \Delta S_{S} 1_{\left\{\left|\Delta S_{s}\right|>\varepsilon\right\}}$, and $S^{\varepsilon}=S-J^{\varepsilon}$. Since $S$ is càdlàg, it has a finite number of jumps larger than any given size, therefore $J^{\varepsilon}$ is piecewise constant and right-continuous. By construction, $S^{\varepsilon}$ is càdlàg with jumps bounded by $\varepsilon$. Since $S$ is càdlàg and $\theta_{-}$left-continuous, by the previous observation $J^{\varepsilon}$ is Stieltjes integrable with respect to $\theta_{-}$. For $S^{\varepsilon}$, consider two partitions $\Gamma=\left(t_{i}\right)_{i=0}^{n}, \Gamma^{\prime}=\left(s_{i}\right)_{i=0}^{m}$, and denote by $\Gamma \cup \Gamma^{\prime}=\left(r_{i}\right)_{i=0}^{l}$. Let $u_{i} \in\left[t_{i-1}, t_{i}\right], v_{i} \in\left[s_{i-1}, s_{i}\right]$ arbitrary points. The corresponding Riemann sums satisfy:

$$
\begin{aligned}
I_{T}\left(S^{\varepsilon}, \theta_{-} ; \Gamma\right)-I_{T}\left(S^{\varepsilon}, \theta_{-} ; \Gamma^{\prime}\right) & =\sum_{t_{i} \leq T} S_{u_{i}}\left(\theta_{t_{i}^{-}}-\theta_{t_{i-1}^{-}}\right)-\sum_{s_{i} \leq T} S_{v_{i}}\left(\theta_{s_{i}^{-}}-\theta_{s_{i-1}^{-}}\right)= \\
& =\sum_{r_{i} \leq T}\left(S_{t\left(r_{i}\right)}-S_{S\left(r_{i-1}\right)}\right)\left(\theta_{r_{i}^{-}}-\theta_{r_{i-1}^{-}}\right)
\end{aligned}
$$

where $t\left(r_{j}\right)=u_{i}$ with $i$ such that $\left[r_{j-1}, r_{j}\right] \subset\left[t_{i-1}, t_{i}\right]$ and $s\left(r_{j}\right)$ is defined analogously. If the meshes of $\Gamma$ and $\Gamma^{\prime}$ are smaller than $\delta$, then $|t(r)-s(r)| \leq \delta$ for all $r$. Applying Lemma A.5 with $s=\varepsilon$ and $\eta=2 \varepsilon$ for some small $\delta$, it follows that:

$$
\left|I_{T}\left(S^{\varepsilon}, \theta_{-} ; \Gamma\right)-I_{T}\left(S^{\varepsilon}, \theta_{-} ; \Gamma^{\prime}\right)\right| \leq \sum_{r_{i} \leq T}\left|S_{t\left(r_{i}\right)}-S_{S\left(r_{i-1}\right)}\right|\left|\theta_{r_{i}^{-}}-\theta_{r_{i-1}^{-}}\right| \leq 2 \varepsilon\|\theta\|_{T}
$$

Since $J^{\varepsilon}$ is Stieltjes integrable, $\left|I_{T}\left(J^{\varepsilon}, \theta_{-} ; \Gamma\right)-I_{T}\left(J^{\varepsilon}, \theta_{-} ; \Gamma^{\prime}\right)\right| \leq 2 \varepsilon\|\theta\|_{T}$ up to a smaller $\delta^{\prime}<\delta$. Adding up, for any $\varepsilon>0$ there is some $\delta^{\prime}>0$ such that:

$$
\left|I_{T}\left(S, \theta_{-} ; \Gamma\right)-I_{T}\left(S, \theta_{-} ; \Gamma^{\prime}\right)\right| \leq 4 \varepsilon\|\theta\|_{T}
$$

for any pair of partitions $\Gamma, \Gamma^{\prime}$ with mesh smaller than $\delta^{\prime}$, which amounts to pathwise integrability in the sense of Stieltjes.

The predictable Stieltjes integral is defined as follows:

Definition A.6 For an arbitrary finite variation $\theta$ and càdlàg $S$, define:

$$
I_{T}(S, \theta):=\int_{[0, T]} S_{u} d \theta_{u}:=\int_{[0, T]} S d \theta_{-}-\sum_{s \leq T}\left(\theta_{s}-\theta_{s^{-}}\right) \Delta S_{S} .
$$

The first term is a well-defined Stieltjes integral by Lemma A.4, and the second term is absolutely convergent:

$$
\sum_{s \leq T}\left(\theta_{s}-\theta_{s^{-}}\right) \Delta S_{s} \leq \sum_{s \leq T}\left|\theta_{s}-\theta_{s-}\right| 2 S_{T}^{*} \leq 2\|\theta\|_{T} S_{T}^{*}<\infty
$$

since $S_{T}^{*}=\sup _{t \in[0, T]}\left|S_{t}\right|$ is finite by the càdlàg assumption. 
Remark A.7 The case for definition (A.2) rests on both mathematical and economic arguments. Proposition A.16 below justifies (A.2) from the viewpoint of stochastic analysis, connecting the predictable Stieltjes integral to the usual stochastic integral via integration by parts.

From the economic viewpoint, consider $S \in \mathscr{D}$ and $\theta \in \mathscr{P}_{V}$, regarding $S$ as the asset price, and $\theta_{t}$ (resp. $\theta_{t-}, \theta_{t^{+}}$) as the number of shares held at time $t$ (resp. immediately before, after $t$ ). Intuitively, a jump of $S$ at a predictable time $\tau$ corresponds to a shock whose size is unknown but whose timing is known before its occurrence (e.g. the announcement of macroeconomic data). Viceversa, a totally inaccessible $\tau$ corresponds to a shock with sudden timing (e.g. a natural catastrophe). The definition of $\int S d \theta$ should reflect the different natures of such jumps.

On a predictable jump, the investor may rebalance her portfolio "immediately" before $\tau$ (and change from position $\theta_{\tau^{-}}$to $\theta_{\tau}$ ) and react after the price has changed from $S_{\tau-}$ to $S_{\tau}$ (by moving from $\theta_{\tau}$ to $\theta_{\tau^{+}}$). On an unpredictable (i.e. $\tau$ totally inaccessible) jump, necessarily $\theta_{\tau^{-}}=\theta_{\tau}$, because $\theta$ is predictable (there is no possibility to prepare for $\tau$ ), but there is still a possibility of rebalancing from $\theta_{\tau}$ to $\theta_{\tau^{+}}$. Thus, the cost of the portfolio $\theta$ should be:

$$
S_{\tau^{-}}\left(\theta_{\tau}-\theta_{\tau^{-}}\right)+S_{\tau}\left(\theta_{\tau^{+}}-\theta_{\tau}\right)=S_{\tau}\left(\theta_{\tau^{+}}-\theta_{\tau^{-}}\right)-\Delta S_{\tau}\left(\theta_{\tau}-\theta_{\tau^{-}}\right)
$$

in the case of predictable $\tau$ and

$$
S_{\tau}\left(\theta_{\tau^{+}}-\theta_{\tau}\right)=S_{\tau}\left(\theta_{\tau^{+}}-\theta_{\tau^{-}}\right)
$$

in the unpredictable case. These formulas are indeed consistent with (A.2). ${ }^{1}$

Looking at (A.3) and (A.4) under a different angle, the predictability of $\theta$ dictates that $\int S d \theta$ should include $\Delta S_{\tau}\left(\theta_{\tau^{+}}-\theta_{\tau}\right)$ at all predictable stopping times, and should not include it at totally inaccessible times.

The pathwise definition of the integral (A.2) extends to a linear map from processes into processes.

Proposition A.8 The function $(S, \theta) \mapsto I .(S, \theta)$

$$
I_{T}(S, \theta)=\int_{[0, T]} S d \theta_{-}-\sum_{s \leq T}\left(\theta_{s}-\theta_{s^{-}}\right) \Delta S_{S}
$$

maps $\mathscr{D} \times \mathscr{P}_{V}$ into $\mathscr{L}$.

Proof It is necessary to check that $\omega \mapsto I_{T}(S, \theta)(\omega)$ is $\mathscr{F}_{T}$-measurable for all $T>0$, and that the paths $t \mapsto I_{t}(S, \theta)$ have right and left limits. For the latter property, observe that the integral in (A.5) is left-continuous with right limits, while the summation in (A.5) is right-continuous with left limits.

For the measurability part, note that the second term in (A.5) is trivially $\mathscr{F}_{T}$ measurable, hence it suffices to check the first term, which is a pathwise Stieltjes

\footnotetext{
1 Note that in the present paper there are also transaction costs represented by the process $\kappa$ hence the additional cost term $\int_{[0, T]} \kappa_{u} d\|\theta\|_{u}$ appears, see Definition 4.4. Remember also that $\int_{[0, T]} S_{t} d \theta_{t}$ (as well as $\left.\int_{[0, T]} \kappa_{u} d\|\theta\|_{u}\right)$ represent the cost of trading with strategy $\theta$ on the interval $[0, T]$, cf. Definition 4.4 .
} 
integral by Lemma A.4. Thus, it is the limit of Riemann sums along a deterministic grid:

$$
\int_{[0, T]} S d \theta_{-}=\lim _{n \rightarrow \infty} \sum_{k=0}^{\lfloor n T\rfloor} S_{\frac{k}{n}}\left(\theta_{\frac{k+1}{n}}{ }^{-}-\theta_{\frac{k}{n}^{-}}\right)
$$

and therefore it is $\mathscr{F}_{T}$-measurable.

The following Theorem establishes the main properties of the predictable Stieltjes integral. First, if $\theta$ is simple (i.e. constant on a sequence of intervals, some of which may collapse to points), then $\int S d \theta$ is consistent with the definition of cost process in the main text. Second, the integral satisfies the natural bound (A.8). Finally, the integral satisfies a dominated convergence theorem and a Fatou property with respect to the pointwise convergence of the integrator $\theta$.

Theorem A.9 The map I in (A.2) satisfies the following properties:

i) If $\left(\tau_{n}\right)_{n \geq 0}$ is a sequence of stopping times, and $\theta=\sum_{n=1}^{\infty}\left(\theta_{\tau_{n}} 1_{\llbracket \tau_{n} \rrbracket}+\theta_{\tau_{n}^{+}} 1_{\rrbracket} \tau_{n}, \tau_{n+1} \llbracket\right)$ is predictable, then:

$$
I_{T}(S, \theta)=\sum_{\tau_{i} \leq T} S_{\tau_{i}^{-}}\left(\theta_{\tau_{i}}-\theta_{\tau_{i}^{-}}\right)+\sum_{\tau_{i}<T} S_{\tau_{i}}\left(\theta_{\tau_{i}^{+}}-\theta_{\tau_{i}}\right)
$$

ii) $I_{T}$ is linear both in $S$ and in $\theta$, and, denoting by $S_{T}^{*}=\sup _{t \in[0, T]}\left|S_{t}\right|$ :

$$
\left|I_{T}(S, \theta)\right| \leq\|\theta\|_{T} S_{T}^{*}
$$

iii) If $\theta^{n} \rightarrow \theta$ pointwise and $\sup _{n>1}\left\|\theta^{n}\right\|_{T}<\infty$, then $I\left(S, \theta^{n}\right) \rightarrow I(S, \theta)$ pointwise.

iv) If $\left(\theta^{n}\right)_{n \geq 1}$ are as in iii) and $S \geq 0$, then $\liminf _{n \rightarrow \infty} I\left(S,\left\|\theta^{n}\right\|\right) \geq I(S,\|\theta\|)$ pointwise.

Proof $i$ ): (A.7) follows immediately from (A.2).

For $i$ ), the linearity in $\theta$ and $S$ is immediate from (A.2), which also implies the estimate:

$$
\left|I_{T}(S, \theta)\right| \leq\left|\int_{[0, T]} S d \theta_{-}\right|+\left|\sum_{t \leq T}\left(\theta_{t}-\theta_{t^{-}}\right) \Delta S_{t}\right| \leq\|\theta\|_{T} S_{T}^{*}+\|\theta\|_{T} 2 S_{T}^{*}=3\|\theta\|_{T} S_{T}^{*}
$$

Now, define the sequence of stopping times $\left(\sigma_{n}^{\varepsilon}\right)_{n \geq 0}$ :

$$
\sigma_{0}^{\varepsilon}=0 \quad \text { and } \quad \sigma_{n+1}^{\varepsilon}=\inf \left\{t>\sigma_{n}^{\varepsilon}:\left|S_{t}-S_{\sigma_{n}^{\varepsilon}}\right|>\varepsilon\right\}
$$

and set $S^{\varepsilon}=\sum_{j=0}^{\infty} S_{\sigma_{j}^{\varepsilon}} 1_{\llbracket \sigma_{j}^{\varepsilon}, \sigma_{j+1}^{\varepsilon} \llbracket}$, which is piecewise constant and right-continuous, and satisfies $\left|S_{t}-S_{t}^{\varepsilon}\right| \leq \varepsilon$ for all $t \in[0, T]$. By linearity in $S$ and (A.9), for any $\varepsilon>0$ the following holds:

$$
\left|I_{T}(S, \theta)\right| \leq\left|I_{T}\left(S^{\varepsilon}, \theta\right)\right|+3 \varepsilon\|\theta\|_{T}
$$


Fix $T>0$, and from now on assume $\theta_{0}=\theta_{s}=0$ for $s \geq T$ without loss of generality. To calculate $I_{T}\left(S^{\varepsilon}, \theta\right)$, first observe that (with the convention $\sigma_{-1}=0$ ):

$$
\begin{gathered}
\int_{[0, T]} S^{\varepsilon} d \theta_{-}=\sum_{n \geq 0} S_{\sigma_{n}}^{\varepsilon}\left(\theta_{\sigma_{n+1}^{-}}-\theta_{\sigma_{n}^{-}}\right)=-\sum_{n \geq 0} \theta_{\sigma_{n}^{-}}\left(S_{\sigma_{n}}-S_{\sigma_{n-1}}\right), \\
\sum_{t \in[0, T]}\left(\theta_{t}-\theta_{t^{-}}\right) \Delta S_{t}^{\varepsilon}=\sum_{n \geq 0}\left(\theta_{\sigma_{n}}-\theta_{\sigma_{n}^{-}}\right)\left(S_{\sigma_{n}}^{\varepsilon}-S_{\sigma_{n}^{-}}^{\varepsilon}\right)=\sum_{n \geq 0}\left(\theta_{\sigma_{n}}-\theta_{\sigma_{n}^{-}}\right)\left(S_{\sigma_{n}}-S_{\sigma_{n-1}}\right) .
\end{gathered}
$$

and therefore:

$$
\begin{aligned}
I\left(S^{\varepsilon}, \theta\right) & =-\sum_{n \geq 0}\left[\theta_{\sigma_{n}^{-}}\left(S_{\sigma_{n}}-S_{\sigma_{n-1}}\right)+\left(\theta_{\sigma_{n}}-\theta_{\sigma_{n}^{-}}\right)\left(S_{\sigma_{n}}-S_{\sigma_{n-1}}\right)\right] \\
& =-\sum_{n \geq 0} \theta_{\sigma_{n}}\left(S_{\sigma_{n}}-S_{\sigma_{n-1}}\right)=\sum_{n \geq 0} S_{\sigma_{n-1}}\left(\theta_{\sigma_{n}}-\theta_{\sigma_{n-1}}\right) .
\end{aligned}
$$

Consequently,

$$
\left|I_{T}\left(S^{\varepsilon}, \theta\right)\right|=\left|\sum_{\sigma_{n} \leq T} S_{\sigma_{n-1}}\left(\theta_{\sigma_{n}}-\theta_{\sigma_{n-1}}\right)\right| \leq S_{T}^{*}\|\theta\|_{T}
$$

and $i$ ) follows since $\varepsilon>0$ is arbitrary.

iii): By the linearity in $S$, it suffices to consider the case of $\theta^{n}$ converging pointwise to zero. $\mathrm{ii}$ ) implies that:

$$
\left|I_{T}\left(S, \theta^{n}\right)\right| \leq\left|I_{T}\left(S^{\varepsilon}, \theta^{n}\right)\right|+\varepsilon\left\|\theta^{n}\right\|_{T}
$$

The calculations in the proof of $i i)$ show that the term $\left|I_{T}\left(S^{\varepsilon}, \theta^{n}\right)\right|$ depends only on finitely many values of $\theta$, thus for large $n$ it becomes arbitrarily small. As $M:=$ $\sup _{n \geq 1}\left\|\theta^{n}\right\|_{T}$ is finite,

$$
\limsup _{n \rightarrow \infty}\left|I_{T}\left(S, \theta^{n}\right)\right| \leq \varepsilon M
$$

and convergence follows since $\varepsilon$ is arbitrary.

$i v)$ : By the same argument as in the proof of Proposition A.1 ii), for $s(\omega)<t(\omega)$ the following holds:

$$
\liminf _{n \rightarrow \infty}\left(\left\|\theta^{n}\right\|_{t}-\left\|\theta^{n}\right\|_{s}\right) \geq\|\theta\|_{t}-\|\theta\|_{s}
$$

in the pointwise sense. Then, recalling (A.11), $S \geq 0$ implies that:

$$
\begin{aligned}
\liminf _{n \rightarrow \infty} I_{T}\left(S^{\varepsilon},\left\|\theta^{n}\right\|\right)=\liminf _{n \rightarrow \infty} & \sum_{k \geq 1} S_{\sigma_{k-1}}\left(\left\|\theta_{\sigma_{k}}^{n}\right\|-\left\|\theta_{\sigma_{k-1}}^{n}\right\|\right) \\
& \geq \sum_{k \geq 1} S_{\sigma_{k-1}}\left(\left\|\theta_{\sigma_{k}}\right\|-\left\|\theta_{\sigma_{k-1}}\right\|\right)=I_{T}\left(S^{\varepsilon},\|\theta\|\right)
\end{aligned}
$$

Since $\left|I_{T}\left(S,\left\|\theta^{n}\right\|\right)-I_{T}\left(S^{\varepsilon},\left\|\theta^{n}\right\|\right)\right| \leq \varepsilon\left\|\theta^{n}\right\|_{T}$ and $\left|I_{T}(S,\|\theta\|)-I_{T}\left(S^{\varepsilon},\|\theta\|\right)\right| \leq \varepsilon\|\theta\|_{T}$, it follows that:

$$
\varepsilon\left(\sup _{n}\left\|\theta_{n}\right\|_{T}+\|\theta\|_{T}\right)+\liminf _{n \rightarrow \infty} \int_{[0, T]} S d\left\|\theta_{n}\right\| \geq \int_{[0, T]} S d\|\theta\| .
$$

and the thesis follows as $\varepsilon \downarrow 0$. 
A.3 Approximations of predictable integrals

The previous subsection shows that the integral $\theta \mapsto I_{T}(S, \theta)$ is continuous with respect to the pointwise convergence. This result is probability free.

A much more precise result holds in a filtered probability space satisfying the usual conditions, if $S$ is locally bounded: both $\theta$ and $I_{T}(S, \theta)$ admit almost surely uniform approximations. Any underlying probability admits an approximating sequence, but this sequence may depend on the probability itself. In this regard, approximating sequence are analogous to announcing sequences for predictable times.

Consider a filtered probability space $\left(\Omega, \mathscr{F},\left(\mathscr{F}_{t}\right)_{t \geq 0}, P\right)$.

Theorem A.10 Let $\theta \in \mathscr{P}_{V}$ and $S \in \mathscr{D}$ be locally bounded. Then, for all $\varepsilon>0$ there exists a strictly increasing sequence of stopping times $\left(\sigma_{n}\right)_{n \geq 0}$ such that $\sup _{n \geq 1} \sigma_{n}>$ $T$, and a predictable process $\theta^{\prime \prime}$ of the form:

$$
\theta^{\prime \prime}=\sum_{n=0}^{\infty}\left(\theta_{\sigma_{n}} 1_{\llbracket \sigma_{n} \rrbracket}+\theta_{\sigma_{n}^{+}} 1_{\rrbracket \sigma_{n}, \sigma_{n+1} \llbracket}\right)
$$

satisfying $\theta^{\prime \prime} \in \mathscr{P}_{V},\left|\theta^{\prime \prime}-\theta\right| \leq \varepsilon,\left|\int S d \theta^{\prime \prime}-\int S d \theta\right| \leq \varepsilon$ and $\left\|\theta^{\prime \prime}\right\| \leq\|\theta\|$ pointwise on $[0, T]$ (outside a P-zero set).

The proof of this theorem requires a property of predictable finite variation processes:

Proposition A.11 Any $\theta \in \mathscr{P}_{V}$ is locally bounded.

Proof Let $\tau_{n}=\inf \left\{t>0:\left|\theta_{t}\right|>n\right\}$, so that $\lim _{n \rightarrow \infty} \tau_{n}=\infty$ a.s. Then $|\theta| 1_{\llbracket 0, \tau_{n} \llbracket}$ is bounded, i.e. $|\theta|$ is prelocally bounded. A prelocally bounded predictable process is also locally bounded by section 11 of Chapter 8 in Dellacherie \& Meyer (1982).

Proof of Theorem A.10. Fix $T>0$. Note that $\theta \in \mathscr{P}_{V}$ implies that $\|\theta\|$ is locally bounded by Proposition A.11, while $S$ is locally bounded by assumption. Thus, there exists a sequence of stopping times $\left(v_{M}\right)_{M \geq 1}, v_{M} \leq T$ such that $\left|S_{t}\right|,\|\theta\|_{t} \leq M$ for $t \in \llbracket 0, v_{M} \rrbracket$, and it suffices to prove the claim on $\llbracket 0, v_{M} \rrbracket$. Indeed, if $\theta^{M}$ satisfies the claim for $\varepsilon^{M}=\varepsilon 2^{-(M+1)}$, then $\theta^{\prime \prime}=\theta^{1} 1_{\llbracket 0, v_{1} \rrbracket}+\sum_{M=2}^{\infty} \theta^{M} 1_{\rrbracket v_{M-1}, v_{M} \rrbracket}$ satisfies the claim on $[0, T]$. Thus, from now on assume that $S=S 1_{\llbracket 0, v_{M} \rrbracket}+S_{v_{M}} 1_{\rrbracket v_{M}, \infty \rrbracket}$. Define the sequence of stopping times $\left(\rho_{m}\right)_{m=0}^{\infty}$ :

$$
\rho_{0}=0 \quad \rho_{m+1}=\inf \left\{t>\rho_{m}:\left|S_{t}-S_{\rho_{m}}\right| \geq \delta\right\} \wedge T .
$$

Since $S$ is càdlàg, $\sup _{m} \rho_{m}>v_{M}$ a.s. on $\left\{v_{M}<T\right\}$.

Step 1: Construction of the approximation on $\rrbracket \rho_{m-1}, \rho_{m} \rrbracket$.

For $m \geq 1$, set $\varepsilon_{m}=\frac{\varepsilon}{2 M} 2^{-(m+1)}$, and define the stopping times $\left(\sigma_{n}\right)_{n \geq 0}$ as follows ${ }^{2}$ :

$$
\begin{aligned}
\sigma_{0} & =\rho_{m-1} \\
v_{0} & =0 \\
\sigma_{n+1} & =\inf \left\{t \geq \sigma_{n}:\|\theta\|_{t} \geq v_{n}+\varepsilon_{m}\right\} \wedge \rho_{m} \\
v_{n+1} & =\inf \left\{\|\theta\|_{t}:\|\theta\|_{t} \geq v_{n}+\varepsilon_{m}, t \leq \rho_{m}\right\}
\end{aligned}
$$

\footnotetext{
${ }^{2}$ The clock $\sigma_{n}$ ticks each time that the total variation $\|\theta\|$ increases by at least $\varepsilon_{m}$, stopping at $\rho_{m}$.
} 
Since $\theta$ has finite variation, the random set $\cup_{n \geq 0} \llbracket \sigma_{n} \rrbracket$ has finite sections. Consider $A_{n} \in \mathscr{F}_{\sigma_{n}}$ such that $\tau_{n}:=\left(\sigma_{n}\right)_{A_{n}}$ is the totally inaccessible part of $\sigma_{n}$. The sequence $\left(\tau_{n}\right)_{n \geq 1}$ is strictly increasing, because $\left(\sigma_{n}\right)_{G_{n}}$ is predictable for $G_{n}:=\left\{\|\theta\|_{\sigma_{n}} \geq\right.$ $\left.v_{n-1}+\varepsilon_{m}\right\}$, hence $A_{n} \subset\left\{\|\theta\|_{\sigma_{n}}<v_{n-1}+\varepsilon_{m},\|\theta\|_{\sigma_{n}^{+}} \geq v_{n-1}+\varepsilon_{m}\right\}$.

The predictable set $B=\cup_{n \geq 0}\left(\llbracket \sigma_{n} \rrbracket \backslash \llbracket \tau_{n} \rrbracket\right)$ admits an exhausting sequence of predictable times $\left(\pi_{n}\right)_{n \geq 0}$. Since $B$ has finite sections, this sequence is strictly increasing up to ordering. ${ }^{3}$ Consider first the process:

$$
\theta^{\prime}=\sum_{n=0}^{\infty}\left(\theta_{\pi_{n}} 1_{\llbracket \pi_{n} \rrbracket}+\theta_{\pi_{n}^{+}} 1_{\rrbracket \pi_{n}, \pi_{n+1} \llbracket}\right)
$$

which is predictable because each $\pi_{n}$ is predictable (cf. Jacod \& Shiryaev (2003, 1.2.12)). Then, set:

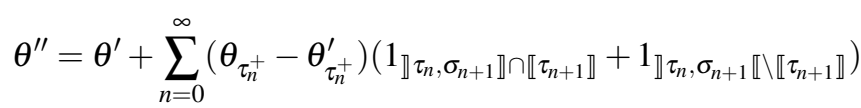

which is again predictable, since $\llbracket \sigma_{n} \rrbracket \backslash \llbracket \tau_{n} \rrbracket \subset \cup_{k \geq 0} \llbracket \pi_{k} \rrbracket$. Thus, $\theta^{\prime}$ constructs an approximation on the accessible parts of $\llbracket \sigma_{n} \rrbracket$, and $\theta^{\prime \prime}$ adjusts it on the totally inaccessible parts of $\llbracket \sigma_{n} \rrbracket$. This two-step procedure is necessary, because the accessible part of $\llbracket \sigma_{n} \rrbracket$ is not identified by a single predictable time. $\theta^{\prime \prime}$ satisfies, for all $n$ :

$$
\begin{array}{r}
\theta_{\pi_{n}^{+}}^{\prime \prime}=\theta_{\pi_{n}^{+}}, \theta_{\tau_{n}^{+}}^{\prime \prime}=\theta_{\tau_{n}^{+}} \\
\theta_{\pi_{n}}^{\prime \prime}=\theta_{\pi_{n}} \\
\theta_{\pi_{n}^{-}}^{\prime \prime}=\theta_{\pi_{n-1}^{+}}, \theta_{\tau_{n}^{-}}^{\prime \prime}=\theta_{\tau_{n-1}^{+}} \\
\theta_{\tau_{n}}=\theta_{\tau_{n}^{-}}, \theta_{\tau_{n}}^{\prime \prime}=\theta_{\tau_{n}^{-}}^{\prime \prime}
\end{array}
$$

(A.19), (A.20) and (A.21) hold by construction, and (A.22) holds because $\tau_{n}$ is totally inaccessible and $\theta, \theta^{\prime \prime}$ are predictable. Finally, note that (A.20) implies that $\theta_{\rho_{m}}=\theta_{\rho_{m}}^{\prime \prime}$ on the accessible part of $\rho_{m}$.

Step 2: Prove the equality:

$$
\int_{\rrbracket \sigma_{n-1}, \sigma_{n} \rrbracket} S d \theta-\int_{\rrbracket \sigma_{n-1}, \sigma_{n} \rrbracket} S d \theta^{\prime \prime}=\int_{\rrbracket \sigma_{n-1}, \sigma_{n} \llbracket} S d \theta-\left(\theta_{\sigma_{n}^{-}}-\theta_{\sigma_{n-1}^{+}}\right)\left(S_{\sigma_{n}^{-}}+\Delta S_{\sigma_{n}} 1_{\llbracket \tau_{n} \rrbracket}\right)
$$

To see this, observe that:

$$
\begin{gathered}
\int_{\rrbracket \sigma_{n-1}, \sigma_{n} \rrbracket} S d \theta=S_{\sigma_{n}^{-}}\left(\theta_{\sigma_{n}}-\theta_{\sigma_{n}^{-}}\right)+S_{\sigma_{n}}\left(\theta_{\sigma_{n}^{+}}-\theta_{\sigma_{n}}\right)+\int_{\rrbracket \sigma_{n-1}, \sigma_{n} \llbracket} S d \theta \\
\int_{\rrbracket \sigma_{n-1}, \sigma_{n} \rrbracket} S d \theta^{\prime \prime}=S_{\sigma_{n}^{-}}\left(\theta_{\sigma_{n}}^{\prime \prime}-\theta_{\sigma_{n}^{-}}^{\prime \prime}\right)+S_{\sigma_{n}}\left(\theta_{\sigma_{n}^{+}}-\theta_{\sigma_{n}}^{\prime \prime}\right)
\end{gathered}
$$

where the second equality exploits (A.19). Hence, the following equality holds:

$$
\int_{\rrbracket \sigma_{n-1}, \sigma_{n} \rrbracket} S d \theta-\int_{\rrbracket \sigma_{n-1}, \sigma_{n} \rrbracket} S d \theta^{\prime \prime}=S_{\sigma_{n}^{-}}\left(\theta_{\sigma_{n}}-\theta_{\sigma_{n}}^{\prime \prime}-\theta_{\sigma_{n}^{-}}+\theta_{\sigma_{n}^{-}}^{\prime \prime}\right)-S_{\sigma_{n}}\left(\theta_{\sigma_{n}}-\theta_{\sigma_{n}}^{\prime \prime}\right)+\int_{\rrbracket \sigma_{n-1}, \sigma_{n} \llbracket} S d \theta
$$

${ }^{3}$ Set $C_{0}=\emptyset, \pi_{n}^{\prime}=\min \left\{t:(\omega, t) \in B \backslash C_{n}\right\}$, and $C_{n}=\cup_{0 \leq k<n} \llbracket \pi_{k}^{\prime} \rrbracket$, which is predictable because $B \backslash C_{n}$ is predictable and $\llbracket \pi_{n}^{\prime} \rrbracket \subset B \backslash C_{n}$, by 1.2.38 of Jacod \& Shiryaev (2003). 
First, recall that $\theta_{\sigma_{n}^{-}}^{\prime \prime}=\theta_{\sigma_{n-1}^{+}}$. On $\llbracket \tau_{n} \rrbracket=\llbracket \sigma_{n} \rrbracket \cap A_{n}$ the first term in the right-hand side vanishes by (A.22), and the claim follows. On $\llbracket \sigma_{n} \rrbracket \backslash \llbracket \tau_{n} \rrbracket$, the second term in the right hand side vanishes by (A.20), while the first one reduces to $S_{\sigma_{n}^{-}}\left(\theta_{\sigma_{n}^{-}}^{\prime \prime}-\theta_{\sigma_{n}^{-}}\right)$.

Step 3: Prove the estimate:

$$
\left|\int_{\rrbracket \rho_{m}, \rho_{m+1} \rrbracket} S d \theta-\int_{\rrbracket \rho_{m}, \rho_{m+1} \rrbracket} S d \theta^{\prime \prime}\right| \leq \delta\left(\|\theta\|_{\rho_{m+1}^{-}}-\|\theta\|_{\rho_{m}^{+}}\right)+\varepsilon 2^{-(m+1)}
$$

First, rewrite (A.23) as:

$$
\int_{\rrbracket \sigma_{n-1}, \sigma_{n} \rrbracket} S d \theta-\int_{\rrbracket \sigma_{n-1}, \sigma_{n} \rrbracket} S d \theta^{\prime \prime}=\int_{\rrbracket \sigma_{n-1}, \sigma_{n} \llbracket}\left(S-S_{\sigma_{n}}^{\prime}\right) d \theta
$$

where $S_{\sigma_{n}}^{\prime}=S_{\sigma_{n}} 1_{\llbracket \tau_{n} \rrbracket}+S_{\sigma_{n}^{-}} 1_{\llbracket \sigma_{n} \rrbracket \llbracket \llbracket \tau_{n} \rrbracket}$. Denote by $N_{m}=\max \left\{n: \sigma_{n}<\rho_{m+1}\right\}$, which implies that $\sigma_{N_{m}+1}=\rho_{m+1}$. By (A.23) it follows that:

$$
\begin{aligned}
& \int_{\rrbracket \rho_{m}, \rho_{m+1} \rrbracket} S d \theta-\int_{\rrbracket \rho_{m}, \rho_{m+1} \rrbracket} S d \theta^{\prime \prime}=\sum_{n=1}^{N_{m}+1}\left(\int_{\rrbracket \sigma_{n-1}, \sigma_{n} \rrbracket} S d \theta-\int_{\rrbracket \sigma_{n-1}, \sigma_{n} \rrbracket} S d \theta^{\prime \prime}\right) \\
= & \left(\sum_{n=1}^{N_{m}} \int_{\rrbracket \sigma_{n-1}, \sigma_{n} \llbracket}\left(S-S_{\sigma_{n}}^{\prime}\right) d \theta\right)+\int_{\rrbracket \sigma_{N_{m}}, \rho_{m+1} \llbracket}\left(S-S_{\rho_{m+1}^{-}}\right) d \theta-\Delta S_{\rho_{m}} 1_{\llbracket \tau_{N_{m}+1} \rrbracket}\left(\theta_{\rho_{m+1}^{-}}-\theta_{\sigma_{N_{m}}^{+}}\right)
\end{aligned}
$$

Now, observe that $\left|S-S_{\rho_{m+1}^{-}}\right| \leq \delta$ and $\left|S-S_{\sigma_{n}}^{\prime}\right| \leq \delta$ for $n \leq N_{m}$, because $\left|S-S_{\rho_{m}}\right| \leq \delta$ on $\llbracket \rho_{m}, \rho_{m+1} \llbracket$. Also, $\left\|\Delta S_{\rho_{m}}\right\| \leq 2 M$ because $|S| \leq M$, and $\left|\theta_{\rho_{m+1}^{-}}-\theta_{\sigma_{N_{m}}^{+}}\right| \leq \varepsilon_{m}=$ $\varepsilon 2^{-(m+1)}$, and the claim follows.

Step 4: Conclusion. Setting $\delta=\varepsilon / M$, and recalling that $\|\theta\|$ is bounded by $M$, it follows that:

$$
\begin{aligned}
\left|\int_{[0, T]} S d \theta-\int_{[0, T]} S d \theta^{\prime \prime}\right| & \leq \sum_{m \geq 1}\left|\int_{\rrbracket \rho_{m-1}, \rho_{m} \rrbracket} S d \theta-\int_{\rrbracket \rho_{m-1}, \rho_{m} \rrbracket} S d \theta^{\prime \prime}\right| \\
& \leq \frac{\varepsilon}{M} \sum_{m \geq 1}\left(\|\theta\|_{\rho_{m}^{-}}-\|\theta\|_{\rho_{m-1}^{+}}\right)+\varepsilon \sum_{m \geq 1} 2^{-(m+1)} \leq 2 \varepsilon
\end{aligned}
$$

The next corollary shows that the interpolation of $\theta$ not only approximates $\int S d \theta$, but any finer Riemann sum.

Corollary A.12 Let $\theta, S,\left(\sigma_{n}\right)_{n \geq 0}$, and $\theta^{\prime \prime}$ be as in Theorem A.10. Let $\left(\tilde{\sigma}_{n}\right)_{n \geq 0}$ be finer than $\left(\sigma_{n}\right)_{n \geq 0}$ (i.e. for all $n \geq 0$, there exists $\tilde{n}(n)$ such that $\sigma_{n}=\sigma_{\tilde{n}(n)}$ a.s.), and define $\tilde{\theta}^{\prime \prime}$ as in (A.12), with $\tilde{\sigma}_{n}$ in the place of $\sigma_{n}$.

Then $\left|\theta^{\prime \prime}-\tilde{\theta}^{\prime \prime}\right| \leq \varepsilon,\left|\int S d \theta^{\prime \prime}-\int S d \tilde{\theta}^{\prime \prime}\right| \leq \varepsilon$ and $\left\|\theta^{\prime \prime}\right\| \leq\left\|\tilde{\theta}^{\prime \prime}\right\|$ pointwise on $[0, T]$ Proof $\left\|\theta^{\prime \prime}\right\| \leq\left\|\tilde{\theta}^{\prime \prime}\right\|$ is trivial. To see that $\left|\theta^{\prime \prime}-\tilde{\theta}^{\prime \prime}\right| \leq \varepsilon$ holds, note that $\tilde{\theta}_{\tilde{\sigma}_{\tilde{n}(n)}^{\prime \prime}}^{\prime \prime}=\theta_{\sigma_{n}}^{\prime \prime}=$ $\theta_{\sigma_{n}}$, and recall that $\|\theta\|$ increases by less than $\varepsilon_{m}$ on $\llbracket \sigma_{n-1}, \sigma_{n} \rrbracket \supset \llbracket \sigma_{\tilde{n}(n-1)}, \sigma_{\tilde{n}(n)} \rrbracket$, whence $\left|\theta^{\prime \prime}-\tilde{\theta}^{\prime \prime}\right| \leq\left|\theta^{\prime \prime}-\theta\right|+\left|\theta-\tilde{\theta}^{\prime \prime}\right| \leq 2 \varepsilon_{m}<\varepsilon$.

To see that $\left|\int S d \theta^{\prime \prime}-\int S d \tilde{\theta}^{\prime \prime}\right| \leq \varepsilon$, observe that the equality (A.23) and the inequality (A.24) continue to hold with $\tilde{\theta}^{\prime \prime}$ in the place of $\theta$, again because $\theta, \theta^{\prime \prime}, \tilde{\theta}^{\prime \prime}$ coincide on $\llbracket \sigma_{n} \rrbracket$. It follows that also the estimate in Step 4 above continues to hold, whence the claim. 
The next corollary is a straightforward consequence of Theorem A.10:

Corollary A.13 Let $\theta \in \mathscr{P}_{V}$ and assume that $(S, \kappa) \in \mathscr{D}$ are locally bounded. Then for all $\varepsilon>0$ there exists $\theta^{\prime \prime}$ as in Proposition A.10, which satisfies $\left|\theta^{\prime \prime}-\theta\right| \leq \varepsilon$, $\left|\int S d \theta^{\prime \prime}-\int S d \theta\right|+\left|\int \kappa d\left\|\theta^{\prime \prime}\right\|-\int \kappa d\|\theta\|\right| \leq \varepsilon$, and $\left\|\theta^{\prime \prime}\right\| \leq\|\theta\|$ pointwise on $[0, T]$.

Repeating the above proof in a deterministic setting yields the following deterministic statement. ${ }^{4}$

Corollary A.14 Let $\theta$ be a finite variation function, and $S$ a càdlàg function. Then, for all $\varepsilon>0$ there exists a sequence of time instants $\left(\tau_{n}\right)_{n \geq 0}$ such that the integrand $\theta^{\prime \prime}$ defined in (A.12) satisfies $\left|\theta^{\prime \prime}-\theta\right| \leq \varepsilon,\left|\int S d \theta^{\prime \prime}-\int S d \theta\right| \leq \varepsilon$ and $\left\|\theta^{\prime \prime}\right\| \leq\|\theta\|$ pointwise.

A simple consequence is that the integral $I$ in (A.2) is the unique extension of the simple integrals (A.7), which satisfies a Lebesgue-type theorem with respect to pointwise convergence of the integrator.

Proposition A.15 The integral (A.2) is the unique extension of (A.7) to the class of all finite variation functions $\theta$ which satisfies iii) in Theorem A.9.

Proof Take $\theta_{n}$ given by Corollary A.14 with the choice $\varepsilon=1 / n$. Let $\tilde{I}$ be an arbitrary extension of the restriction of $I$ to simple $\theta$ which satisfies $i i i)$ of Theorem A.9. Then necessarily $\tilde{I}(S, \theta)=\lim _{n \rightarrow \infty} I\left(S, \theta_{n}\right)$, and this latter limit is $I(S, \theta)$, by Corollary A.14.

When $S$ is a semimartingale, integration by parts links the predictable Stieltjes integral to the usual stochastic integral:

Proposition A.16 Let $\theta \in \mathscr{P}_{V}$ and $S$ a càdlàg semimartingale. Then:

$$
\int_{0}^{T} S_{u} d \theta_{u}=\theta_{T} S_{T}-\theta_{0} S_{0}-\int_{0}^{T} \theta_{u} d S_{u}
$$

where the first integral is in the predictable Stieltjes sense and the second one is a usual stochastic integral.

Proof Without loss of generality, assume $\theta_{0}=\theta_{T}=0$. Then the stochastic integral exists, as $\theta$ is locally bounded by Proposition A.11. Consider first $\theta$ simple. By linearity of the integrals, it suffices to treat the left- and right-continuous cases separately, and localization allows us to assume $\|\theta\|, \theta$ bounded.

First, consider $\theta$ left-continuous with finitely many jumps $\left(\tau_{j}\right)_{0 \leq j \leq n}$. Then:

$$
I_{T}(S, \theta)=\sum_{j=0}^{n} S_{\tau_{j}}\left(\theta_{\tau_{j+1}}-\theta_{\tau_{j}}\right)=-\sum_{j=0}^{n} \theta_{\tau_{j}}\left(S_{\tau_{j}}-S_{\tau_{j-1}}\right)=-\int_{0}^{T} \theta_{u} d S_{u}
$$

by the definition of (elementary) stochastic integrals, with the convention $\tau_{-1}=0$. This argument shows the statement for $\theta$, and extends easily to arbitrary simple leftcontinuous $\theta$.

\footnotetext{
4 But a pathwise application of Corollary A.14 does not prove Proposition A.10, which requires the predictability of $\theta^{\prime \prime}$.
} 
Take now $\theta$ right-continuous. Without loss of generality, suppose (see the proof of Theorem A.10) that the jump times $\tau_{j}$ are predictable with announcing sequences $\tau_{j-1} \leq \tau_{j}^{k}<\tau_{j}, k \geq 1$. Set

$$
\gamma(k):=\sum_{j=0}^{\infty}\left(\theta_{\tau_{j}} 1_{\rrbracket \tau_{j}, \tau_{j+1}^{k} \rrbracket}+E\left(\theta_{\tau_{j+1}} \mid \mathscr{F}_{\tau_{j+1}^{k}}\right) 1_{\rrbracket \tau_{j+1}^{k}, \tau_{j+1} \rrbracket}\right) .
$$

Note that $\theta_{\tau_{j+1}}$ is $\mathscr{F}_{\tau_{j+1}{ }^{-}}$-measurable (see 1.2.4 of Jacod \& Shiryaev (2003)), hence the conditional expectation converges a.s. to $\theta_{\tau_{j+1}}$ as $k \rightarrow \infty$, by the martingale convergence theorem. Hence $\gamma(k) \rightarrow \theta$ pointwise (possibly outside a $P$-zero set).

It follows from the previous step, by the dominated convergence theorem for stochastic integrals, and by Theorem A.9 iii) that

$$
-\int_{0}^{T} \theta_{u} d S_{u}=\lim _{k \rightarrow \infty}-\int_{0}^{T} \gamma_{u}(k) d S_{u}=\lim _{k \rightarrow \infty} I(S, \gamma(k))=I(S, \theta) .
$$

For general $\theta \in \mathscr{P}_{V}$, take an approximation $\theta^{n}$ constructed in Theorem A.10 with $\varepsilon:=1 / n$. The above arguments imply that $I_{T}\left(S, \theta^{n}\right)=-\int_{0}^{T} \theta_{u}^{n} d S_{u}$ for all $n$. $I_{T}\left(S, \theta^{n}\right) \rightarrow I_{T}(S, \theta)$ by Theorem A.10 and the stochastic integrals of $\theta^{n}$ converge to that of $\theta$ by dominated convergence (note that $\left|\theta_{n}\right| \leq|\theta|+1 / n$ ).

\section{B Appendix}

For completeness, this appendix recalls the statements of some now classical tools in Mathematical Finance. First, a compactness lemma for bounded sets in $L_{+}^{0}$ :

Lemma B.1 (Delbaen \& Schachermayer (1994, Lemma A1.1)) Let $\left(f_{n}\right)_{n \geq 1}$ be a sequence of $[0, \infty)$-valued measurable functions on a probability space $(\Omega, \mathscr{F}, P)$. There exists a sequence $g_{n} \in \operatorname{conv}\left(\left(f_{k}\right)_{k \geq n}\right)$ such that $\left(g_{n}\right)_{n \geq 1}$ converges almost surely to a $[0, \infty]$-valued function $g$. If $\operatorname{conv}\left(\left(f_{n}\right)_{n \geq 1}\right)$ is bounded in $L^{0}$, then $g$ is finite almost surely.

Second, an important consequence of the Krein-Smulian Theorem (cf. Delbaen \& Schachermayer (1994), Kabanov \& Last (2002, Lemma 4.4)):

Lemma B.2 Let $C \subset L^{\infty}$ be a convex set. Then $C$ is $\sigma\left(L^{\infty}, L^{1}\right)$-closed if and only if $C \cap\left\{Z:\|Z\|_{\infty} \leq x\right\}$ is closed in probability for all $x>0$.

Third, a version of the Kreps (1981)-Yan (1980) separation theorem (cf. Schachermayer (1994) or Kabanov \& Stricker (2001b)):

Theorem B.3 Let $-L_{+}^{\infty} \subset C \subset L^{\infty}$ be a convex cone, closed in the $\sigma\left(L^{\infty}, L^{1}\right)$-topology, such that $C \cap L_{+}^{\infty}=\{0\}$. Then there exists a probability $Q$ equivalent to $P$ such that $E_{Q}[C] \leq 0$.

Finally, a compactness lemma for finite variation processes (cf. Guasoni (2002, Lemma 3.4), Campi \& Schachermayer (2006, Proposition 14)). Note that Lemma A.2 allows to avoid section theorems.

Lemma B.4 Consider $\left(\theta^{n}\right)_{n \geq 1} \subset \mathscr{P}_{V}$ such that $\left(\left\|\theta^{n}\right\|_{T}\right)_{n \geq 1}$ is bounded in $L^{0}$. Then there exists $\theta \in \mathscr{P}_{V}$ and a sequence $\left(\eta^{n}\right)_{n \geq 1} \subset \mathscr{P}_{V}$ of convex combinations $\eta^{n} \in$ $\operatorname{conv}\left(\theta^{n}, \theta^{n+1}, \ldots\right)$ converging to $\theta$ pointwise such that also $\left\|\eta_{n}\right\| \rightarrow\|\eta\|$ pointwise. 


\section{References}

Black, F. \& Scholes, M. (1973), 'The Pricing of Options and Corporate Liabilities', The Journal of Political Economy 81(3), 637-654.

Campi, L. \& Schachermayer, W. (2006), 'A super-replication theorem in Kabanov's model of transaction costs', Finance Stoch. 10(4), 579-596.

Cherny, A. (2007), General arbitrage pricing model. II. Transaction costs, in 'Séminaire de Probabilités XL', Vol. 1899 of Lecture Notes in Math., Springer, Berlin, pp. 447-461.

Choulli, T. \& Stricker, C. (1998), Séparation d'une sur- et d'une sousmartingale par une martingale, in 'Séminaire de Probabilités, XXXII', Vol. 1686 of Lecture Notes in Math., Springer, Berlin, pp. 67-72.

Dalang, R. C., Morton, A. \& Willinger, W. (1990), 'Equivalent martingale measures and no-arbitrage in stochastic securities market models', Stochastics Stochastics Rep. 29(2), 185-201.

Delbaen, F. \& Schachermayer, W. (1994), 'A general version of the fundamental theorem of asset pricing', Math. Ann. 300(3), 463-520.

Delbaen, F. \& Schachermayer, W. (2006), The mathematics of arbitrage, Springer Finance, Springer-Verlag, Berlin.

Dellacherie, C. \& Meyer, P.-A. (1978), Probabilities and potential, Vol. 29 of NorthHolland Mathematics Studies, North-Holland Publishing Co., Amsterdam.

Dellacherie, C. \& Meyer, P.-A. (1982), Probabilities and potential. B, Vol. 72 of North-Holland Mathematics Studies, North-Holland Publishing Co., Amsterdam. Theory of martingales, Translated from the French by J. P. Wilson.

Dybvig, P. \& Ross, S. (1987), 'Arbitrage', The New Palgrave: A Dictionary of Economics 1, 100-106.

Guasoni, P. (2002), 'Optimal investment with transaction costs and without semimartingales', Ann. Appl. Probab. 12(4), 1227-1246.

Guasoni, P., Rásonyi, M. \& Schachermayer, W. (2010), 'The fundamental theorem of asset pricing for continuous processes under small transaction costs', Annals of Finance 6(2), 157-191.

Harrison, J. M. \& Kreps, D. M. (1979), 'Martingales and arbitrage in multiperiod securities markets', J. Econom. Theory 20(3), 381-408.

Harrison, J. M. \& Pliska, S. R. (1981), 'Martingales and stochastic integrals in the theory of continuous trading', Stochastic Process. Appl. 11(3), 215-260.

Jacod, J. \& Shiryaev, A. N. (2003), Limit theorems for stochastic processes, Vol. 288 of Grundlehren der Mathematischen Wissenschaften [Fundamental Principles of Mathematical Sciences], second edn, Springer-Verlag, Berlin.

Jouini, E. \& Kallal, H. (1995), 'Martingales and arbitrage in securities markets with transaction costs', J. Econom. Theory 66(1), 178-197.

Kabanov, Y. (1999), 'Hedging and liquidation under transaction costs in currency markets', Finance Stoch. 3(2), 237-248.

Kabanov, Y. M. \& Last, G. (2002), 'Hedging under transaction costs in currency markets: a continuous-time model', Math. Finance 12(1), 63-70.

Kabanov, Y. M. \& Stricker, C. (2001a), 'The Harrison-Pliska arbitrage pricing theorem under transaction costs', J. Math. Econom. 35(2), 185-196. Arbitrage and 
control problems in finance.

Kabanov, Y., Rásonyi, M. \& Stricker, C. (2002), 'No-arbitrage criteria for financial markets with efficient friction', Finance Stoch. 6(3), 371-382.

Kabanov, Y. \& Stricker, C. (2001b), A teachers' note on no-arbitrage criteria, in 'Séminaire de Probabilités, XXXV', Vol. 1755 of Lecture Notes in Math., Springer, Berlin, pp. 149-152.

Kramkov, D. O. (1996), 'Optional decomposition of supermartingales and hedging contingent claims in incomplete security markets', Probab. Theory Related Fields 105(4), 459-479.

Kreps, D. M. (1981), 'Arbitrage and equilibrium in economies with infinitely many commodities', J. Math. Econom. 8(1), 15-35.

Rásonyi, M. (2003), A remark on the superhedging theorem under transaction costs, in 'Séminaire de Probabilités XXXVII', Vol. 1832 of Lecture Notes in Math., Springer, Berlin, pp. 394-398.

Ross, S. A. (1977), Return, risk and arbitrage., in I. Friend \& J. Bicksler, eds, 'Risk and Return in Finance', Ballinger, Cambridge, MA.

Schachermayer, W. (1994), 'Martingale measures for discrete-time processes with infinite horizon', Math. Finance 4(1), 25-55.

Schachermayer, W. (2004), 'The fundamental theorem of asset pricing under proportional transaction costs in finite discrete time', Math. Finance 14(1), 19-48.

Wheeden, R. L. \& Zygmund, A. (1977), Measure and integral, Marcel Dekker Inc., New York. An introduction to real analysis, Pure and Applied Mathematics, Vol. 43.

Yan, J. A. (1980), Caractérisation d'une classe d'ensembles convexes de $L^{1}$ ou $H^{1}$, in 'Seminar on Probability, XIV (Paris, 1978/1979) (French)', Vol. 784 of Lecture Notes in Math., Springer, Berlin, pp. 220-222. 